\title{
New $Y$ and T Dwarfs from WISE Identified by Methane Imaging ${ }^{* \dagger}$
}

\author{
C. G. Tinney ${ }^{1,2}$ (1) , J. Davy Kirkpatrick ${ }^{3}$, Jacqueline K. Faherty ${ }^{4}\left(\mathbb{1}\right.$, Gregory N. Mace $^{5}\left(\mathbb{D}\right.$, Mike Cushing $^{6}\left(\mathbb{0}\right.$, Christopher R. Gelino $^{3}$, \\ Adam J. Burgasser ${ }^{7}$ (D), Scott S. Sheppard ${ }^{8}(\mathbb{D})$, and Edward L. Wright ${ }^{9}$ (D) \\ ${ }^{1}$ Exoplanetary Science at UNSW, School of Physics, UNSW Sydney, NSW 2052, Australia; c.tinney@unsw.edu.au \\ ${ }^{2}$ Australian Centre for Astrobiology, UNSW Sydney, NSW 2052, Australia \\ ${ }^{3}$ IPAC, Mail Code 100-22, Caltech, Pasadena, CA 91125, USA \\ ${ }^{4}$ Department of Astrophysics, American Museum of Natural History, New York, NY 10023, USA \\ ${ }^{5}$ McDonald Observatory and Department of Astronomy, University of Texas at Austin, Austin, TX 78712-1205, USA \\ ${ }^{6}$ Department of Physics and Astronomy, The University of Toledo, OH 43606, USA \\ ${ }^{7}$ Center for Astrophysics and Space Science, University of California San Diego, La Jolla, CA 92093, USA \\ ${ }^{8}$ Department of Terrestrial Magnetism, Carnegie Institution for Science, Washington, DC 20015, USA \\ 9 Department of Physics and Astronomy, UCLA, Los Angeles, CA 90095-1547, USA \\ Received 2017 December 27; revised 2018 March 26; accepted 2018 March 27; published 2018 May 17
}

\begin{abstract}
We identify new Y-and T-type brown dwarfs from the WISE All Sky data release using images obtained in filters that divide the traditional near-infrared $\mathrm{H}$ and $\mathrm{J}$ bands into two halves-specifically $\mathrm{CH}_{4} \mathrm{~S}$ and $\mathrm{CH}_{4} \mathrm{l}$ in the $\mathrm{H}$ and $\mathrm{J} 2$, and $\mathrm{J} 3$ in the $\mathrm{J}$. This proves to be very effective at identifying cool brown dwarfs via the detection of their methane absorption, as well as providing preliminary classification using methane colors and WISE -to-nearinfrared colors. New and updated calibrations between T/Y spectral types and $\mathrm{CH}_{4} \mathrm{~s}-\mathrm{CH}_{4} 1 \mathrm{~J} 3-\mathrm{W} 2$, and $\mathrm{CH}_{4} \mathrm{~s}-\mathrm{W} 2$ colors are derived, producing classification estimates good to a few spectral sub-types. We present photometry for a large sample of $\mathrm{T}$ and $\mathrm{Y}$ dwarfs in these filters, together with spectroscopy for 23 new ultra-cool dwarfs-2 $\mathrm{Y}$ dwarfs and $21 \mathrm{~T}$ dwarfs. We identify a further 8 new cool brown dwarfs, which we have high confidence are $\mathrm{T}$ dwarfs based on their methane photometry. We find that, for objects observed on a $4 \mathrm{~m}$ class telescope at J-band magnitudes of $\sim 20$ or brighter, $\mathrm{CH}_{4} \mathrm{~s}-\mathrm{CH}_{4} \mathrm{l}$ is the more powerful color for detecting objects and then estimating spectral types. Due to the lower sky background in the J-band, the J3 and J2 bands are more useful for identifying fainter cool dwarfs at $J \gtrsim 22$. The J3-J2 color is poor at estimating spectral types. But fortunately, once J3-J2 confirms that an object is a cool dwarf, the J3-W2 color is very effective at estimating approximate spectral types.
\end{abstract}

Key words: brown dwarfs - methods: observational - techniques: photometric

\section{Introduction}

Data from the NASA Wide-field Infrared Survey Explorer (WISE; Wright et al. 2010; Mainzer et al. 2011) have delivered unprecedented advances in our understanding of the properties and space densities of the coldest compact astrophysical sources identified outside our solar system-the T- and Y-type brown dwarfs (e.g., Cushing et al. 2011; Kirkpatrick et al. 2011, 2012, 2013). These very cold brown dwarfs have scientific impacts that span multiple astronomical arenas. In the field of star formation, they can deliver a historical record of the star formation process at very low masses and at epochs billions of years prior to the star-forming regions we observe today. In the field of planetary atmospheric theory, they represent low-temperature atmospheres that can be readily observed without the contaminating glare of a host star, and without the photochemical complications introduced by host star irradiation. In the field of exoplanet searches, they provide nearby, low-luminosity search targets that potentially host planetary systems of their own, which could have implications for the debate on what differentiates a low-mass brown dwarf from a high-mass planet.

WISE readily identifies these very cold brown dwarfs as a result of their strong thermal-infrared methane absorption. The shortest-wavelength WISE band (hereafter W1) has a central

\footnotetext{
* This paper includes data gathered with the $3.9 \mathrm{~m}$ Anglo-Australian Telescope located at Siding Spring Observatory, Coonabarabran, Australia.

$\dagger$ This paper includes data gathered with the $6.5 \mathrm{~m}$ Magellan Telescopes located at Las Campanas Observatory, Chile.
}

wavelength of $3.4 \mu \mathrm{m}$, which sits in the middle of the strong fundamental methane absorption band near $3.3 \mu \mathrm{m}$. The second shortest WISE band (hereafter W2), has a central wavelength of $4.6 \mu \mathrm{m}$, where the photosphere is reasonably transparent and thus detects flux from deeper, hotter layers in the brown dwarf. As a result, cold brown dwarfs can be identified via their very red W1-W2 color. WISE's relatively uniform all-sky coverage, coupled with its ability to identify cool objects even when quite close to the Galactic plane, makes it the ideal data source from which to generate a complete thermal-infrared magnitudelimited sample of T and Y dwarfs in the Solar Neighborhood. The generation of just such a $\mathrm{T} / \mathrm{Y}$ dwarf census is a key goal of the WISE Science Team brown dwarf collaboration. Large numbers of Y-and T-type brown dwarfs have been identified and spectroscopically confirmed to date by the WISE Science Team brown dwarf collaboration using color selection (see, e.g., Cushing et al. 2011; Kirkpatrick et al. 2011, 2012; Mainzer et al. 2011; Tinney et al. 2012; Mace et al. 2013), and more recently, proper motion selection from the AllWISE and NEOWISE samples (see, e.g., Kirkpatrick et al. 2014, 2016; Schneider et al. 2016b). Additional cool brown dwarfs have been found by multiple teams independently working with public WISE data (e.g., Luhman 2014; Pinfield et al. 2014), while others have been identified from searches for cool companions to nearby stars companions to nearby stars (e.g., Luhman et al. 2011; Liu et al. 2012; Dupuy et al. 2015).

The WISE Science Team brown dwarf collaboration has largely selected targets for follow-up on the basis of photometry, consistent with $\mathrm{W} 1-\mathrm{W} 2>2.9$ over the full range 
Table 1

AAT $\mathrm{CH}_{4} \mathrm{~s}, \mathrm{CH}_{4} 1$ Methane Imaging

\begin{tabular}{|c|c|c|c|}
\hline Run & Prog. & Useful Nights & Seeing \\
\hline 2011 Jun $11-18$ & $11 \mathrm{~A} / 14$ & $\cdots$ & $\ldots$ \\
\hline 2011 Sep 6-13 & $11 \mathrm{~B} / 26$ & Sep $6,7,10,12,13$ & $1 . " 05-1.5$ \\
\hline 2012 May 30-Jun 3 & $12 \mathrm{~A} / 27$ & May 30,31 & $1 " .2-2$ ". 0 \\
\hline 2012 Jun $27-30$ & $12 \mathrm{~A} / 27$ & Jun $28,29,30$ & $1 ", 2-2 " .0$ \\
\hline 2012 Sep 4-6 & $12 \mathrm{~B} / 26$ & Sep $4,5,6$ & $1 " .4-2 "$ "2 \\
\hline 2012 Dec 28-Jan 1 & $12 \mathrm{~B} / 26$ & Dec $28,29,30,31$, Jan 1 & 1 ". $2-2$ ". 6 \\
\hline
\end{tabular}

of WISE W2 magnitudes, plus somewhat bluer objects (i.e., down to $\mathrm{W} 1-\mathrm{W} 2=2.0$ ) if colors and magnitudes suggested a distance less than $20 \mathrm{pc}$. In many cases these objects are nondetections at $\mathrm{W} 1$ (i.e., their $\mathrm{W} 1-\mathrm{W} 2$ colors are $3 \sigma$ upper limits). This substantially increases the observational phase space probed by this follow-up program, at the cost of increasing the number of "false positives" identified from the WISE data, which must then be eliminated by subsequent observations.

Follow-up observations require the identification and spectral classification of a near-infrared counterpart to the WISE source. Unfortunately, the coldest objects (i.e., the Y dwarfs) can be very faint in the near-infrared (i.e., $J \sim H \gtrsim 22$ ), making them challenging targets even for $8 \mathrm{~m}$ class ground-based telescopes. T dwarfs in WISE will be somewhat brighter in the near-infrared, and this gives medium-sized $4 \mathrm{~m}$ class telescopes a role in targeting them as part of the completion of the WISE $\mathrm{T} / \mathrm{Y}$ dwarf census.

Methane imaging can play a useful role in this process. The discovery of very strong and broad methane absorption in the spectrum of the known T dwarf (Gl 229B Nakajima et al. 1995; Oppenheimer et al. 1995) made use of this spectral signature for $\mathrm{T}$ dwarf identification using specially designed imaging filters self-evidently obvious. This was confirmed when Rosenthal et al. (1996) used a circularly variable filter (i.e., an adjustable narrowband filter) to differentially re-detect Gl 229B. Subsequent use of methane imaging (rather than spectroscopy) to "winnow" out $\mathrm{T}$ dwarfs from wide-field survey data was first carried out by Herbst et al. (1999), while the first use of methane filters in blind searches for new T dwarfs was carried out by Mainzer \& Ian (2003). At around the same time, Tinney et al. (2005) began using specially constructed methane filters in the IRIS2 instrument at the Anglo-Australian Telescope to identify $\mathrm{T}$ dwarfs from lists of candidates from the 2MASS survey.

The power of this technique for WISE follow-up comes from the fact that the identification of a near-infrared counterpart that shows methane absorption within a small distance on the sky from a WISE All-Sky or AlLWISE position uniquely identifies this near-infrared object as the counterpart to the WISE source. Following this, the strength of the methane absorption can then provide a spectral type estimate, reducing the necessity for spectroscopic follow-up in all cases.

\section{Imaging-Anglo-Australian Telescope (AAT)}

Imaging observations were carried out on the AAT on the nights listed in Table 1 with the IRIS2 imaging spectrograph (Tinney et al. 2005). IRIS2 is a near-infrared imager and spectrograph, with a single $1024 \times 1024$ pixel detector giving an imaging field of view of $7 ! 7$ on a side at a pixel scale of 0 ". 4486/pixel.

Data were obtained in seeing conditions ranging from 1"!05 to over 2." 0 . Targets for observation were selected in 2011 from then extant catalogs using the pipeline software developed to produce the WISE Preliminary Data Release (for details see the Explanatory Supplement ${ }^{10}$ ), and in 2012 from the WISE AllSky release. As the optimal candidate selection procedures were being developed at the same time as improved versions of the WISE pipeline, we report in Table 2 the WISE photometry for our observed sources as presented in the WISE All-Sky data release made in 2012 March (rather than the photometry on which their original selections were based).

In general, the selected candidates satisfy the criteria that they were detected in $\mathrm{W} 2$ and had $\mathrm{W} 1-\mathrm{W} 2<2.0$. The WISE processing pipeline records either detections at greater than $3 \sigma$ significance, or $3 \sigma$ upper limits. Requiring a W1 detection (or $3 \sigma$ upper limit) and $\mathrm{W} 1-\mathrm{W} 2<2.0$, pushes the effective W2 detection floor to be much brighter than that imposed by the pipeline's $3 \sigma$ detection threshold. Our faintest $\mathrm{T}$ and $\mathrm{Y}$ dwarf candidates typically had $\mathrm{W} 2 \lesssim 15.5$, which corresponds to a signal-to-noise ratio of $\sim 10$. Additional selection criteria have been found to greatly assist in improving the rejection of non-brown dwarf contaminants. Broadly, we require that the source was not identified as an artifact (i.e., known to be spurious), and that it was not flagged as blended (in which case it would have poorly determined photometry). For more details, including values of the specific flags used, see Section 2.1 of Kirkpatrick et al. (2012).

\subsection{J-band Imaging}

Each candidate was initially observed in the J-band with a planned exposure time of 54 minutes, broken up into thirty-six 90s pseudo-randomly dithered exposures. Online data reduction using the ORACDR pipeline system (Cavanagh et al. $2008)^{11}$ would then produce a near-publication-quality processed sub-mosaic soon after the first nine images of this dither pattern were completed. This processed image was then analyzed using purpose-built Perl scripts that automated the extraction of photometry for the image (using SExtractor; Bertin \& Arnouts 1996), followed by the photometric and astrometric calibration of that data using the 2MASS Point Source Catalog (PSC; Skrutskie et al. 2006).

This meant that within 5 minutes of the completion of the first 9-image sub-mosaic, we would know whether a J-band positional counterpart had been identified down to $J \approx 19.5$. If a positionally matched candidate clearly emerged after this first sub-mosaic (when such an object emerged, it usually did so at the $<10-\sigma$ level), then the dithering sequence would be truncated, and methane imaging observations would begin. In the absence of an "early" match, the J-band imaging sequence was allowed to run to completion, providing imaging data to a depth of $J \approx 21$.

\subsection{Methane Imaging}

Once a plausible J-band counterpart had been identified, methane imaging observations were carried out with the $\mathrm{CH}_{4} \mathrm{~S}$ and $\mathrm{CH}_{4} 1$ filters in IRIS2. The use of these filters for the study

\footnotetext{
${ }^{10}$ http://wise2.ipac.caltech.edu/docs/release/prelim/expsup/

${ }^{11}$ See also http://www.oracdr.org/.
} 
Table 2

WISE , $J_{\mathrm{MKO}}$ and $\mathrm{CH}_{4} \mathrm{~s}-\mathrm{CH}_{4}$ lphotometry for Candidate Cool WISE Brown Dwarfs

\begin{tabular}{|c|c|c|c|c|c|c|c|c|c|c|c|}
\hline WISE Desig. ${ }^{a}$ & W1 & W2 & $\mathrm{W} 1-\mathrm{W} 2$ & $J_{\mathrm{MKO}}{ }^{\mathrm{b}}$ & $\mathrm{CH}_{4} \mathrm{~s}^{\mathrm{c}}$ & $\mathrm{CH}_{4} \mathrm{~s}-\mathrm{CH}_{4} \mathrm{l}^{\mathrm{c}}$ & $\mathrm{CH}_{4} \mathrm{SpT}^{\mathrm{d}}$ & $J-\mathrm{W} 2$ & $\mathrm{CH}_{4} \mathrm{~S}-\mathrm{W} 2$ & Spec & Notes \\
\hline \multicolumn{12}{|c|}{$\mathrm{T} / \mathrm{Y}$ dwarfs : Position matches with unresolved sources showing $\mathrm{CH}_{4}$ absorption } \\
\hline J001505.87-461517.6 & 17.020 .15 & 14.250 .06 & 2.770 .16 & 17.760 .06 & 17.450 .06 & -1.240 .16 & T7.0 0.5 & 3.510 .09 & 3.230 .08 & $\mathrm{~T} 8$ & FIRE, this paper \\
\hline J003231.09-494651.4 & 18.080 .34 & 15.070 .09 & 3.010 .35 & 18.540 .09 & 18.200 .07 & -1.960 .23 & T8.6 0.5 & 3.470 .13 & 3.130 .11 & $\mathrm{~T} 8.5$ & FIRE, this paper \\
\hline J014807.25-720258.7 & $>18.94$ & 14.690 .05 & $>4.25$ & $\ldots$ & 18.770 .05 & -2.340 .20 & T9.2 0.5 & $\ldots$ & 4.080 .07 & T9.5 & Kirkpatrick et al. (2012) \\
\hline J024124.73-365328.0 & 16.890 .10 & 14.340 .04 & 2.550 .11 & 16.590 .04 & 16.550 .03 & -1.060 .06 & T6.5 0.5 & 2.250 .06 & 2.210 .08 & $\mathrm{~T} 7$ & FIRE, this paper \\
\hline J030919.67-501614.3 & 16.510 .08 & 13.610 .03 & 2.900 .09 & 17.170 .03 & 17.010 .03 & -1.310 .06 & $\mathrm{~T} 7.20 .5$ & 3.560 .10 & 3.400 .05 & $\ldots$ & \\
\hline J032504.33-504400.3 & $>18.73$ & 15.700 .10 & $>3.03$ & 18.940 .09 & 18.390 .09 & -2.200 .36 & T9.0 0.5 & 3.240 .13 & 2.690 .13 & T9 & Schneider et al. (2015) \\
\hline J035000.32-565830.2 & $>18.90$ & 14.730 .06 & $>4.17$ & 22.470 .49 & $\ldots$ & $\ldots$ & $\ldots$ & 7.740 .51 & $\ldots$ & Y1 & Kirkpatrick et al. (2012) \\
\hline J040443.48-642029.9 & $>18.86$ & 15.730 .09 & $>2.60$ & 19.550 .22 & 19.430 .04 & -1.270 .10 & T7.1 0.5 & 3.820 .23 & 3.700 .10 & T9 & Schneider et al. (2015) \\
\hline J041022.71+150248.4 & $>18.33$ & 14.180 .06 & $>4.15$ & $\ldots$ & 20.470 .07 & $\ldots$ & $\ldots$ & $\ldots$ & 6.290 .09 & Y0 & Kirkpatrick et al. (2012) \\
\hline J062842.71-805725.0 & $>18.78$ & 15.450 .08 & $>3.32$ & 18.710 .16 & 18.340 .04 & $\begin{array}{lll}-2.30 & 0.13\end{array}$ & T9.1 0.5 & 3.260 .18 & 2.890 .09 & $\ldots$ & \\
\hline J064528.38-030248.2 & $>18.18$ & 14.940 .10 & $>3.24$ & 16.910 .04 & 16.880 .04 & -1.000 .09 & T6.3 0.5 & 1.970 .12 & 1.940 .15 & $\mathrm{~T} 6$ & SpeX, this paper \\
\hline J071322.55-291751.9 & $>18.35$ & 14.480 .06 & $>3.87$ & 19.640 .15 & 19.330 .04 & -2.720 .15 & T9.7 0.5 & 5.160 .16 & 4.850 .08 & Y0 & Kirkpatrick et al. (2012) \\
\hline J071301.84-585445.1 & $>19.04$ & 15.440 .07 & $>3.60$ & $\ldots$ & $\ldots$ & $\ldots$ & $\ldots$ & $\ldots$ & $\ldots$ & T9 & FIRE, this paper \\
\hline J072227.27-054029.9 & 15.190 .05 & 12.210 .03 & 2.980 .06 & 16.520 .02 & 16.390 .02 & -2.120 .04 & T8.9 0.5 & 4.310 .03 & 4.180 .03 & $T 9^{f}$ & Kirkpatrick et al. (2012) \\
\hline J091408.96-345941.5 & $>17.83$ & 15.030 .09 & $>2.80$ & 18.360 .11 & 17.790 .02 & -1.700 .07 & T8.2 0.5 & 3.330 .14 & 2.760 .10 & $\mathrm{~T} 8$ & FIRE, this paper \\
\hline J094020.10-220820.5 & 17.010 .14 & 14.570 .07 & 2.430 .16 & 17.360 .05 & 17.300 .04 & -1.520 .11 & T7.8 0.5 & 2.790 .17 & 2.730 .08 & $\mathrm{~T} 8$ & SpeX, this paper \\
\hline J105553.59-165216.3 & $>18.37$ & 15.040 .10 & $>3.33$ & 20.790 .02 & 20.350 .06 & $<-1.69$ & $>\mathrm{T} 8.2$ & 5.750 .11 & 5.310 .14 & $\mathrm{~T} 9$ & FIRE, this paper \\
\hline J111239.24-385700.7 & 17.970 .40 & 14.360 .06 & 3.610 .40 & 20.260 .15 & 19.940 .05 & -1.720 .12 & T8.2 0.5 & 5.900 .16 & 5.580 .08 & T9 & FIRE, this paper \\
\hline J114156.71-332635.8 & 17.200 .17 & 14.530 .06 & 2.670 .18 & 19.760 .14 & 19.690 .04 & $\left(\begin{array}{lll}-0.71 & 0.09\end{array}\right)^{\mathrm{g}}$ & $\left(\begin{array}{lll}\mathrm{T} 5 & 0.5\end{array}\right)^{\mathrm{g}}$ & 5.230 .15 & 5.160 .07 & Y0 & FIRE, this paper \\
\hline J143311.42-083736.4 & $>18.74$ & 15.230 .10 & $>3.51$ & 19.070 .11 & $\ldots$ & $\ldots$ & $\ldots$ & 3.840 .15 & $\ldots$ & $\mathrm{T} 8^{\mathrm{h}}$ & FIRE, this paper \\
\hline J144806.48-253420.3 & $>18.28$ & 15.030 .09 & $>3.25$ & 18.850 .11 & 18.710 .13 & -1.020 .29 & T6.4 0.5 & 3.820 .14 & 3.680 .16 & $\mathrm{~T} 8$ & FIRE, this paper \\
\hline J150115.92-400418.4 & 16.480 .11 & 14.210 .05 & 2.070 .12 & 16.530 .04 & 16.050 .03 & -0.860 .06 & T5.9 0.5 & 2.320 .06 & 1.840 .05 & T6 & SpeX, this paper \\
\hline $\mathrm{J} 173551.72-820900.1$ & 15.610 .06 & 13.730 .04 & 1.880 .07 & 16.580 .03 & 16.410 .03 & -0.760 .08 & T5.6 0.5 & 2.850 .05 & 2.680 .05 & $\mathrm{~T} 6$ & FIRE, this paper \\
\hline $\mathrm{J} 201748.72-342102.5$ & $>18.21$ & 15.090 .13 & $>3.12$ & 20.890 .24 & 20.190 .06 & -1.460 .15 & T7.7 0.5 & 5.800 .27 & 5.100 .14 & $\ldots$ & \\
\hline $\mathrm{J} 205628.91+145953.2$ & $>18.25$ & 13.930 .05 & $>4.33$ & $\ldots$ & 18.990 .04 & -3.170 .25 & Y0.2 0.5 & $\ldots$ & 5.060 .06 & Y0 & Kirkpatrick et al. (2011) \\
\hline J210200.15-442919.5 & 16.940 .17 & 14.120 .05 & 2.830 .18 & 18.250 .06 & 18.060 .06 & -2.200 .24 & Т9.0 0.5 & 4.130 .08 & 3.940 .08 & $\mathrm{~T} 9$ & NIRSPEC, this paper \\
\hline J215949.48-480854.9 & 17.760 .29 & 14.540 .07 & 3.190 .30 & 18.890 .08 & 18.640 .09 & $\begin{array}{lll}-1.31 & 0.28\end{array}$ & $\mathrm{~T} 7.20 .5$ & 4.350 .11 & 4.100 .13 & T9 & FIRE, this paper \\
\hline $\mathrm{J} 221140.52-475826.5$ & 17.670 .21 & 14.580 .06 & 3.090 .22 & 17.380 .04 & 17.380 .04 & -1.510 .14 & T7.8 0.5 & 2.800 .07 & 2.800 .07 & $\ldots$ & \\
\hline J221216.33-693121.6 & 18.050 .40 & 14.900 .09 & 3.150 .41 & 19.720 .13 & 19.290 .04 & -3.150 .23 & Y0.2 0.5 & 4.820 .16 & 4.390 .10 & T9.5 & FIRE, this paper \\
\hline J222055.31-362817.4 & $>18.65$ & 14.660 .06 & $>3.99$ & 20.380 .17 & 20.200 .07 & $<-1.52$ & $>\mathrm{T} 7.8$ & 5.720 .18 & 5.580 .09 & Y0 & Kirkpatrick et al. (2012) \\
\hline J223204.50-573010.5 & $>17.90$ & 15.150 .11 & $>2.75$ & 18.860 .09 & 18.740 .10 & -1.490 .30 & T7.7 0.5 & 3.710 .14 & 3.590 .14 & $\mathrm{~T} 9$ & FIRE, this paper \\
\hline $\mathrm{J} 230228.68-713441.6$ & 17.140 .14 & 14.280 .05 & 2.860 .14 & 16.970 .04 & 17.520 .07 & -1.140 .15 & T4.7 0.5 & 2.690 .06 & 3.240 .09 & $\ldots$ & \\
\hline J233226.49-432510.6 & $>17.88$ & 14.990 .09 & $>2.89$ & $\ldots$ & 19.140 .04 & $<-2.59$ & $>\mathrm{T} 9.5$ & $\ldots$ & 4.150 .09 & T9: & NIRSPEC, this paper \\
\hline J235425.33-564928.6 & 16.930 .13 & 14.840 .08 & 2.090 .15 & 17.090 .04 & 16.980 .04 & -0.550 .07 & T7.8 0.5 & 2.250 .09 & 2.140 .09 & T6 & FIRE, this paper \\
\hline J235447.80-814044.9 & 18.230 .33 & 15.030 .07 & $>3.20$ & 17.640 .05 & 17.680 .06 & -1.040 .12 & T6.4 0.5 & 2.610 .09 & 2.650 .10 & $\ldots$ & \\
\hline \multicolumn{12}{|c|}{$\mathrm{T} / \mathrm{Y}$ dwarfs : Position matches $\left(<1^{\prime \prime}\right)$ with an extended source, sources showing no methane absorption and/or non-detections } \\
\hline J012102.92-190656.9 & 16.470 .09 & 14.710 .07 & 1.760 .11 & 19.670 .16 & 18.870 .13 & +0.170 .17 & $\ldots$ & 4.960 .17 & 4.160 .15 & $\ldots$ & $\mathrm{i}$ \\
\hline J071939.54-173514.8 & 16.720 .07 & 13.710 .03 & 3.010 .08 & $\ldots$ & 20.10 .1 & +0.330 .15 & $\ldots$ & $\ldots$ & 6.390 .17 & $\ldots$ & $\mathrm{j}$ \\
\hline J074551.79-015122.1 & 18.140 .41 & 14.970 .09 & 3.170 .42 & 19.900 .16 & 19.530 .15 & +0.250 .20 & $\ldots$ & 4.930 .17 & 4.560 .17 & $\ldots$ & $\mathrm{i}$ \\
\hline J083942.85-402938.9 & 17.370 .24 & 13.660 .04 & 3.710 .25 & 20.60 .2 & 18.820 .11 & +0.400 .15 & $\ldots$ & 6.940 .22 & 5.160 .12 & $\ldots$ & $\mathrm{k}$ \\
\hline $\mathrm{J} 130740.45-463035.1$ & 15.000 .03 & 12.930 .03 & 2.070 .04 & $\ldots$ & 16.590 .04 & +0.240 .05 & $\ldots$ & $\ldots$ & 3.660 .05 & $\ldots$ & 1 \\
\hline $\mathrm{J} 150711.06-344026.0$ & 15.640 .06 & 14.030 .05 & 1.620 .08 & 17.730 .04 & 17.180 .05 & +0.260 .07 & $\ldots$ & 3.700 .07 & 3.150 .09 & $\ldots$ & $\mathrm{m}$ \\
\hline J164445.19-645628.9 & $>18.50$ & 15.220 .10 & $>3.28$ & $\ldots$ & 18.130 .09 & +0.150 .11 & $\ldots$ & $\ldots$ & 2.910 .13 & $\ldots$ & $\mathrm{i}$ \\
\hline J185709.40-315345.5 & 16.480 .12 & 14.420 .07 & 2.060 .14 & 18.580 .10 & 17.840 .09 & +0.050 .12 & $\ldots$ & 4.160 .12 & 3.420 .11 & $\ldots$ & $\mathrm{i}$ \\
\hline J190230.27-371246.1 & 17.180 .21 & 14.460 .07 & 2.720 .22 & 20.270 .29 & $\ldots$ & $\ldots$ & $\ldots$ & 5.810 .30 & $\ldots$ & $\ldots$ & $\mathrm{n}$ \\
\hline J193441.70-490837.6 & 17.000 .12 & 14.500 .06 & 2.500 .13 & 20.360 .31 & $\ldots$ & $\ldots$ & $\ldots$ & 5.860 .32 & $\ldots$ & $\ldots$ & See Table 4 \\
\hline J203020.25-692043.0 & 18.060 .43 & 14.830 .09 & 3.230 .44 & 19.510 .17 & 18.830 .15 & -0.070 .23 & $\ldots$ & 4.680 .20 & 4.000 .17 & $\ldots$ & o $\quad-2-3$ \\
\hline
\end{tabular}


Table 2

(Continued)

\begin{tabular}{|c|c|c|c|c|c|c|c|c|c|c|c|}
\hline WISE Desig. ${ }^{a}$ & W1 & W2 & $\mathrm{W} 1-\mathrm{W} 2$ & $J_{\mathrm{MKO}}{ }^{\mathrm{b}}$ & $\mathrm{CH}_{4} \mathrm{~s}^{\mathrm{c}}$ & $\mathrm{CH}_{4} \mathrm{~s}-\mathrm{CH}_{4} \mathrm{l}^{\mathrm{c}}$ & $\mathrm{CH}_{4} \mathrm{SpT}^{\mathrm{d}}$ & $J-\mathrm{W} 2$ & $\mathrm{CH}_{4} \mathrm{~S}-\mathrm{W} 2$ & Spec & Notes \\
\hline J203119.30-690500.3 & $>17.58$ & 14.410 .07 & $>3.17$ & 20.440 .22 & 19.180 .03 & -0.410 .06 & T4.2 0.5 & 6.030 .23 & 4.770 .08 & $\cdots$ & $\mathrm{p}$ \\
\hline J224245.85-201511.0 & $>18.32$ & 15.150 .13 & $>3.17$ & 20.030 .16 & $\ldots$ & $\ldots$ & $\ldots$ & 4.880 .21 & $\ldots$ & $\ldots$ & q \\
\hline
\end{tabular}

Notes.

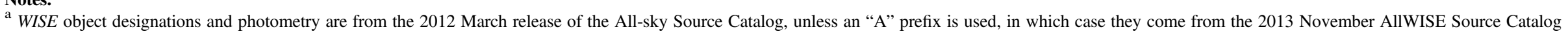

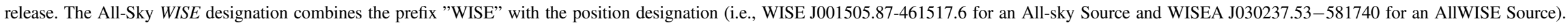
These are abbreviated with the first four digits of the right ascension and declination throughout this paper-e.g., W0015-4615.

${ }^{\mathrm{b}}$ IRIS2 J photometry was obtained through a $J_{\text {МКо }}$ filter, differentially calibrated using $J_{2 \mathrm{MASS}}$ photometry converted to $J_{\text {Мко }}$ in each field.

${ }^{c}$ IRIS2 $\mathrm{CH}_{4} \mathrm{~S}$ and $\mathrm{CH}_{4} \mathrm{~s}-\mathrm{CH}_{4}$ lphotometry calibrated (as described in the text) on the system of Tinney et al. (2005).

${ }^{\mathrm{d}}$ Estimated spectral types using $\mathrm{CH}_{4} \mathrm{~s}-\mathrm{CH}_{4} \mathrm{l}$ and the calibration of Section 6.1.

${ }^{\mathrm{e}}$ The discovery name for this object is UGPS J072227.51-054031.2 (Lucas et al. 2010) and $J_{\mathrm{MKO}}$ is from that paper.

${ }^{\mathrm{f}}$ While noting that the spectral type for this object has been the subject of some debate, we adopt the T9 type of Kirkpatrick et al. (2012).

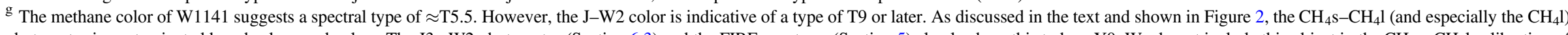

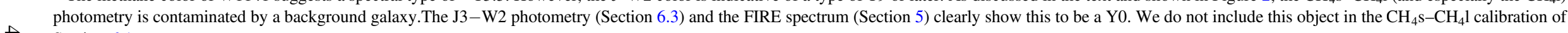
Section 6.1.

h 143311.42-083736.4: a spectrum and spectral type for this object have also been published by Lodieu et al. (2012). Those values are in agreement with those presented here.

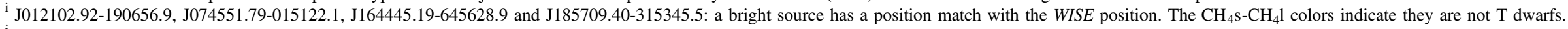

${ }^{\mathrm{j}} \mathrm{J}$ 071939.54-173514.8: extended source at position with no methane absorption.

${ }^{\mathrm{k}}$ J083942.85-402938.9: is clearly an extended source $\left(2 !^{\prime \prime} 1 \times 3\right.$ !" $\left.^{\prime}\right)$, with no methane absorption. WISE source is likely this galaxy.

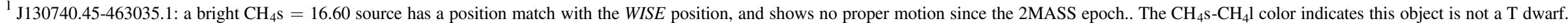

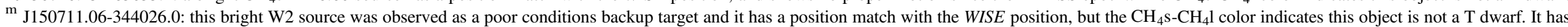
shown no proper motion since the 2MASS epoch).

${ }^{\mathrm{n}} \mathrm{J} 190230.27-371246.1$ : very extended (3"!5) source at position, making this galaxy the likely WISE source.

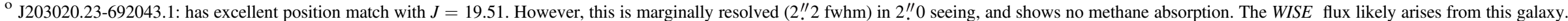

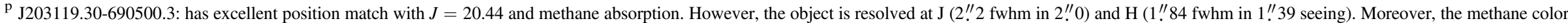

suggests a spectral type of $\mathrm{T} 4$, which is inconsistent with the $\mathrm{J}-\mathrm{W} 2$ and $\mathrm{CH}_{4} \mathrm{~s}-\mathrm{W} 2$ colors (which suggest a much later spectral type of $>\mathrm{T} 9$ ). This identification is therefore considered tentative.

${ }^{q}$ J224245.85-201511.0: has $J=20.03$ object 1!'2 from the WISE position. However, this object has almost identical J and J2 magnitudes, which suggests methane absorption would be extremely weak. 
Table 3

Magellan J2/J3 Methane Imaging

\begin{tabular}{lc}
\hline \hline UT Date & Median Seeing $\left(^{\prime \prime}\right)$ \\
\hline 2012 Mar 10 & 0.64 \\
2012 May 10 & 0.56 \\
2012 Jul 6 & 0.70 \\
2012 Jul 7 & 0.53 \\
2013 Jan 15 & 0.40 \\
2013 Mar 22 & 0.56 \\
2013 Apr 22 & 0.57 \\
2013 Aug 15 & 0.50 \\
2016 Nov 18 & 0.80 \\
2017 Jan 05 & 0.54 \\
\hline
\end{tabular}

of cool brown dwarfs is described in Tinney et al. (2005)—we summarize just the salient points here. The IRIS2 methane filters $\left(\mathrm{CH}_{4} \mathrm{~S}\right.$ and $\left.\mathrm{CH}_{4} \mathrm{l}\right)$ divide the $\mathrm{H}$-band in half, sampling the wavelength ranges $1.520-1.620 \mu \mathrm{m}$ and $1.640-1.740 \mu \mathrm{m}$ (respectively). Flux in the $\mathrm{CH}_{4} 1$ filter is substantially depressed by methane absorption in $\mathrm{T}$ and $\mathrm{Y}$ dwarfs, so the $\mathrm{CH}_{4} \mathrm{~S}-\mathrm{CH}_{4} \mathrm{l}$ color provides a powerful means of determining $\mathrm{T}$ and $\mathrm{Y}$ dwarf classifications. Methane filter observations are obtained by interleaving $\mathrm{CH}_{4} \mathrm{~S}$ and $\mathrm{CH}_{4} \mathrm{l}$ observations (in an ABBA sense), while also dithering the telescope on the sky. These data are also processed "on the fly" by ORACDR and deliver pairs of reduced images after every 7-9 pairs of images, which are then processed and differentially calibrated onto the $\mathrm{CH}_{4} \mathrm{~S}-\mathrm{CH}_{4} \mathrm{l}$ photometric system of Tinney et al. (2005). As with the J-band imaging, purpose-built scripts are run as soon as the first pair of $\mathrm{CH}_{4} \mathrm{~S}$ and $\mathrm{CH}_{4} \mathrm{l}$ mosaics are produced. Once again, if a clear methane signature was detected, the observing sequence was truncated, and observations moved onto the next WISE candidate.

\subsection{Photometry}

Following the initial processing done at the telescope, the ORACDR pipeline was used to reprocess these data for final analysis. A simple two-step process is followed: in the first step, all images on each object are processed together to produce a flat-field and a first-pass mosaic, allowing the flattened images that went into this first-pass mosaic to be analyzed to determine their photometric zero-point and image quality; in the second-pass, frames with poor image quality or poor photometric throughput are culled and the remaining images were reprocessed to produce a final mosaic. Photometry was then obtained from the final mosaics on each field, by differentially calibrating onto the $J_{\mathrm{MKO}}$ or $\mathrm{CH}_{4} \mathrm{~s}-\mathrm{CH}_{4} \mathrm{l}$ systems using 2MASS PSC photometry for stars lying within the IRIS2 field of view.

For J-band data this differential calibration is achieved by identifying objects in the field of view with 2 MASS $-0.2<$ $J-K<1.5$ and then converting those $\mathrm{J}$ magnitudes from the 2MASS photometric system to the MKO photometric system, using the equations determined by J. Carpenter in the 2MASS All-sky Survey Explanatory Supplement. ${ }^{12}$ As the IRIS2 J,H, $\mathrm{K}, \mathrm{Ks}$ filters are MKO ones (i.e., manufactured to the prescriptions of Tokunaga et al. 2002), the result is $\mathbf{J}$ photometry on the MKO system.

\footnotetext{
12 http://www.ipac.caltech.edu/2mass/releases/allsky/doc/sec6_4b.html
}

For $\mathrm{CH}_{4} \mathrm{~s}, \mathrm{CH}_{4} \mathrm{l}$ data, objects in the field of view for which transformations from the 2MASS to MKO photometric systems are reliable (i.e., have $2 \mathrm{MASS}$ colors in ranges where the Carpenter transformations are valid-see Tinney et al. 2005) are converted to $\mathrm{MKO} \mathrm{H}$, and then used to define zero-points for the $\mathrm{CH}_{4} \mathrm{~S}$ and $\mathrm{CH}_{4} \mathrm{l}$ systems, and to determine $\mathrm{CH}_{4} \mathrm{~s}-\mathrm{CH}_{4} \mathrm{l}$. The resulting zero-points for a given field are typically determined to be between $\pm 0.02-0.1 \mathrm{mag}$, and the scatter about the zero-point determination is used to determine a standard error in the mean, which is then propagated to the finally quoted photometric uncertainties.

Table 2 presents $\mathrm{J}$ and $\mathrm{CH}_{4} \mathrm{~s}-\mathrm{CH}_{4} \mathrm{l}$ photometry from the AAT, as well as WISE W1 and W2 photometry from the AllSky release-the official WISE designation for all sources combines the prefix "WISE" with the position designation in column 1 of the table-e.g., WISE J001505.87-461517.6. These are abbreviated thereafter with the letter "W" and the first four digits of the right ascension and declination of the designation-e.g., W0015-4615. Also listed in the table are spectral types resulting from spectroscopic observations in this paper (Section 5) and other programs, as well as estimated spectral types based on a new $\mathrm{CH}_{4} \mathrm{~s}-\mathrm{CH}_{4} \mathrm{l}$ calibration derived in Section 6.1.

\section{Imaging-Magellan}

Imaging observations were obtained using the FourStar imaging camera (Persson et al. 2013) on the Magellan Baade telescope between 2012 March 10 and 2017 January 5. FourStar is a near-infrared mosaic imager with four $2048 \times 2048$ pixel detectors giving an imaging field of view of $11^{\prime}$ on a side at a pixel scale of 0 ". $159 /$ pixel. It is equipped with a set of intermediate-band filters, originally specified for the measurement of photometric redshifts. These filters turn out to be almost ideally suited for observing very cool brown dwarfs (see Figure 1 in Tinney et al. 2012). In particular, the J3 filter $\left(\lambda_{\text {cen }} \approx 1.29 \mu \mathrm{m}, 90 \%\right.$ of the peak throughput range $1.210-1.366 \mu \mathrm{m})$, collects almost all of the "methane free" $\mathrm{J}$-band flux from late $\mathrm{T}$ and $\mathrm{Y}$ dwarfs, while the $\mathrm{J} 2$ filter $\left(\lambda_{\text {cen }} \approx 1.14 \mu \mathrm{m}, 90 \%\right.$ of the peak throughput range 1.067$1.224 \mu \mathrm{m})$ is strongly impacted by methane absorption between $1.1 \mu \mathrm{m}$ and $1.2 \mu \mathrm{m}$. All the observations described here were performed with the WISE target positioned in FourStar's Chip 2.

Image quality over the course of this program varied between 0.4 and 0.7 (see Table 3). Our observing and analysis techniques follow those previously described by us (Tinney et al. 2012, 2014), and involve observing each target with the FourStar J3 and J2 filters in a sequence of 60-20s pseudo-randomly dithered exposures. Targets are observed with net integration times ranging from 5 minutes to $1.0 \mathrm{~h}$. As with the AAT data, images are processed at the telescope using a modified version of the ORACDR ${ }^{13}$ data reduction pipeline, and examined during observing to determine whether a counterpart had been detected. As for the AAT data, the final analysis involves running ORACDR twice for each jitter set, with individual exposures of poor image quality removed from the list used in the second-pass.

Photometric processing and calibration followed the procedures outlined above for AAT data, except for the final stage of calibration onto the $\mathrm{J} 2 / \mathrm{J} 3$ photometric system, which followed

\footnotetext{
${ }^{13}$ http://www.ukirt.hawaii.edu/instruments/cgs4/orac-dr/printable.html
} 
Table 4

WISE and Magellan J3,J2 photometry of Candidate Cool Brown Dwarfs from WISE

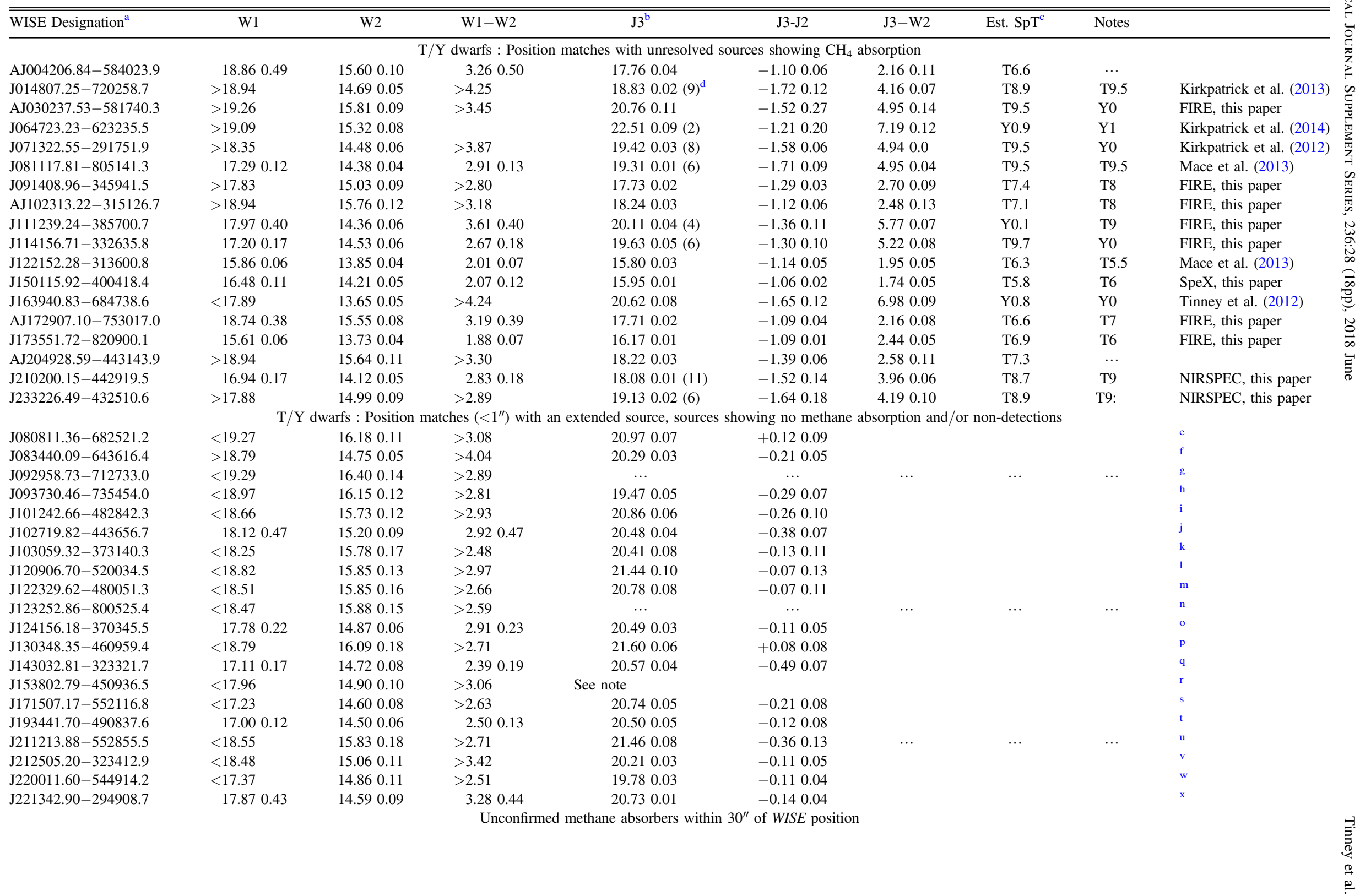


$\checkmark \quad{ }^{k} \mathrm{~J} 103059.32-373140.3$ : object with no methane absorption 1"! 9 from a WISE position. No methane absorbers within $30^{\prime \prime}$ to $33=21$.

${ }^{1} \mathrm{~J} 120906.70-520034.5$ : object with no methane absorption 0" " 5 from a WISE position. No methane absorbers within $30^{\prime \prime}$ to J3 = 21 .

${ }^{\mathrm{m}} \mathrm{J} 122329.62-480051.3$ : object with no methane absorption 1 ." 5 from a WISE position. No methane absorbers within $30^{\prime \prime}$ to $\mathrm{J} 3=21.5$.

"J123252.86-800525.4: no sources within 10" of a WISE position to $J=21$. No methane absorbers within $30^{\prime \prime}$.

o J124156.18-370345.5: galaxy (resolved in 0!" 48 seeing) 0!" 3 from a WISE position. No methane absorbers within $30^{\prime \prime}$ to J3 = 22.5 .

p J130348.35-460959.4: galaxy $\left(1.0 \times 0\right.$ ". 8 in $0{ }^{\prime \prime} 55$ seeing $)$ at a WISE position. No methane absorbers within $30^{\prime \prime}$ to J3 $=22.5$.

${ }^{\mathrm{q}} \mathrm{J} 143032.81-323321.7$ : galaxy (resolved in 0". 47 seeing) 0". 3 from a WISE position. No methane absorbers within $30^{\prime \prime}$ to $\mathrm{J} 3=22$

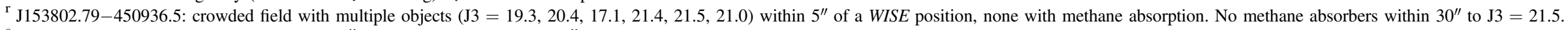

s J171507.17-552116.8: unresolved source (0!" 6 seeing) with no methane 0 " 5 from WISE . No methane absorbers within $30^{\prime \prime}$ to J3 = 22.5 .

${ }^{\mathrm{t}} \mathrm{J} 193441.70-490837.6: \mathrm{J} 3=20.5$ galaxy ( 1 !' 1 resolved in 0 "! 9 seeing) 0 "! 2 from WISE . No methane absorbers within $30^{\prime \prime}$ to J3 = 21.5 .

" J211213.88-552855.5: object 2!" 4 from a WISE position with no methane absorption. No methane absorbers within $30^{\prime \prime}$ to J3 = 21.5.

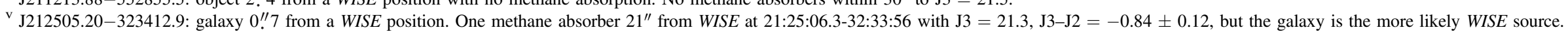

" J220011.60-544914.2: galaxy (1!"0 resolved in 0". 6 seeing) 0". 4 from a WISE position. No methane absorbers within $30^{\prime \prime}$ to J3=22.

${ }^{x} \mathrm{~J} 221342.90-294908.7$ : galaxy $\left(0.7 \times 00^{\prime \prime} 6\right)$ 0!" 3 from a WISE position with no methane. No methane absorbers within $30^{\prime \prime}$ to J3 $=22$.

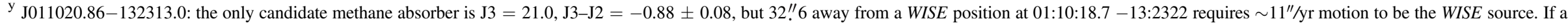
match would have $\mathrm{J} 3-\mathrm{W} 2=6.17$ suggesting a possibly $\mathrm{Y} 0$.

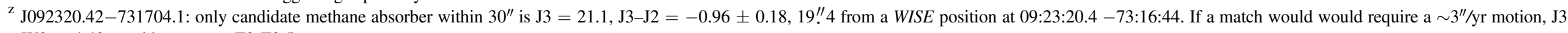
$-\mathrm{W} 2=4.42$ would suggest a T9-T9.5.

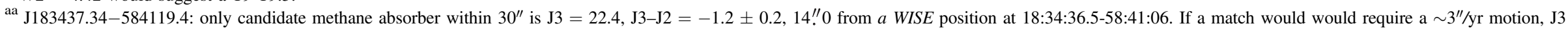

$-\mathrm{W} 2=6.8 \pm 0.2$ would suggest a Y0. 

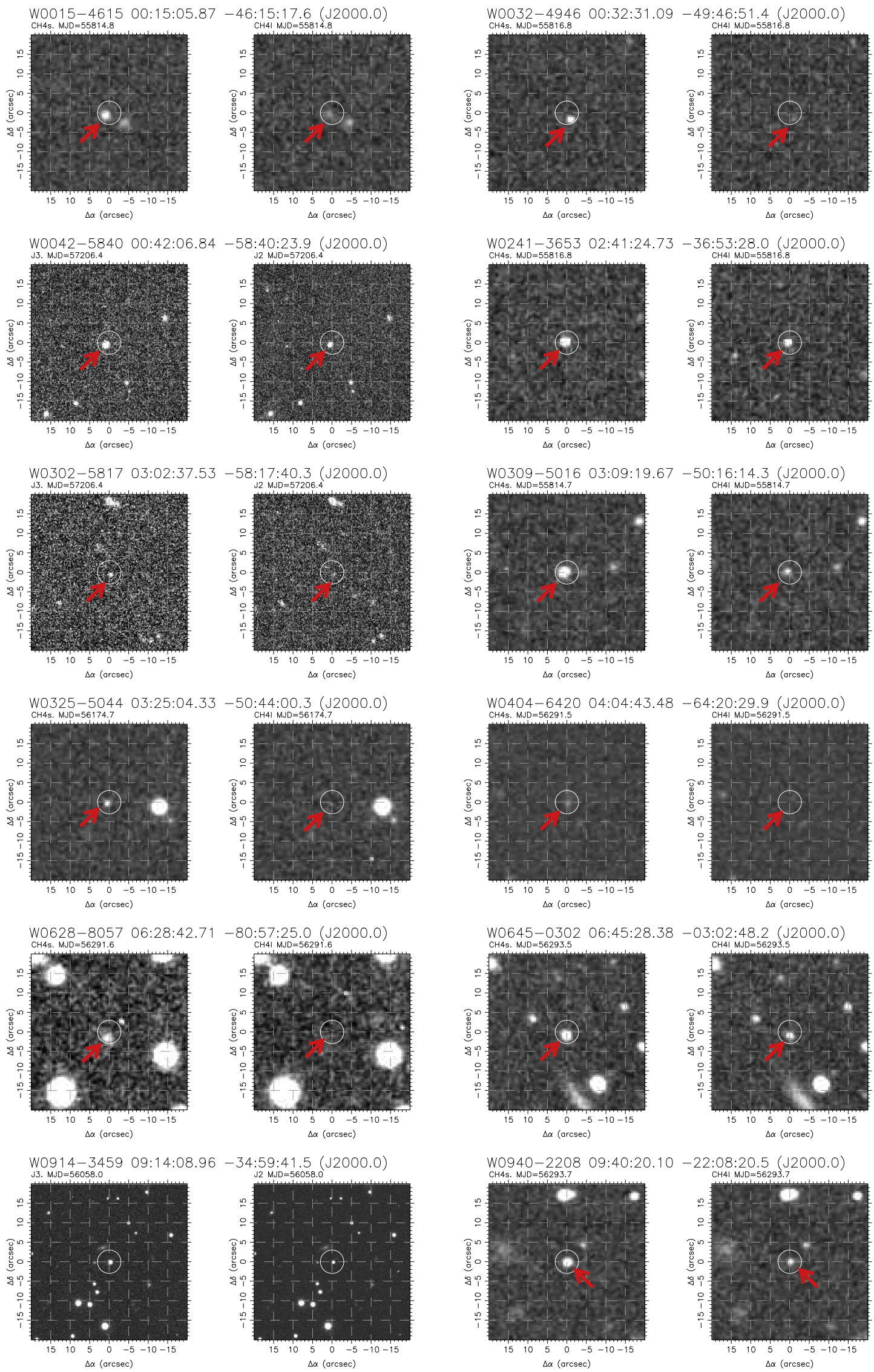

Figure 1. (a) Pairs of finding charts in $\mathrm{CH}_{4} \mathrm{~S} / \mathrm{CH}_{4} 1$ or $\mathrm{J} 3 / \mathrm{J} 2$ for the imaging observations tabulated in Tables 2 and 4 . All charts are $40^{\prime \prime}$ on a side, with north to the top and west to the right, centered on the WISE source position, and with a $3^{\prime \prime}$ circle drawn at the WISE position. Methane-absorbing objects are brighter in the left panel of each pair of images. In ambiguous cases, an arrow is used to identify the cool brown dwarf. (b) The single image of W1433-0837 is a J-band image from the AAT, with the arrow indicating the object observed spectroscopically.) 

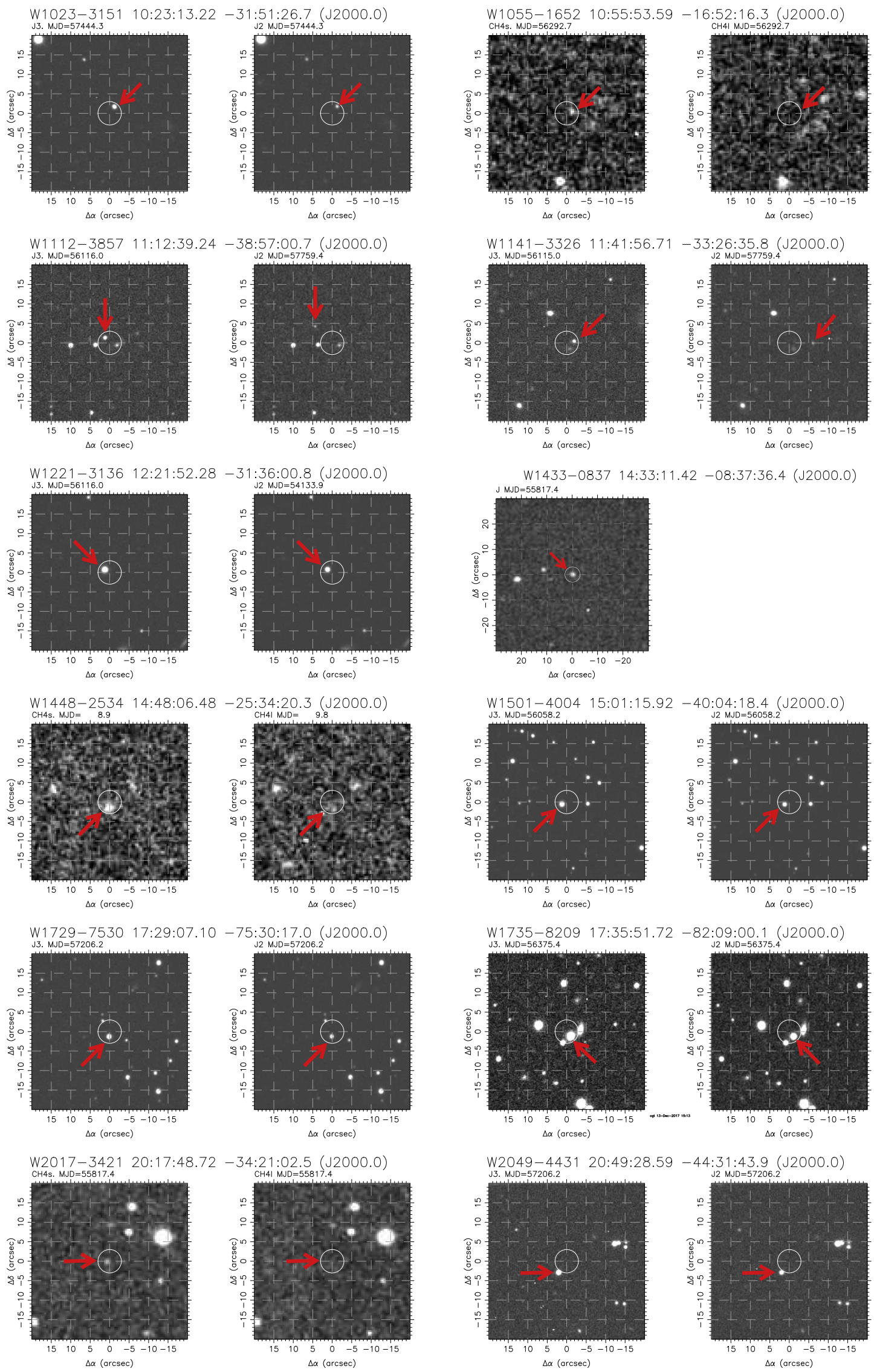

Figure 1. (Continued.) 

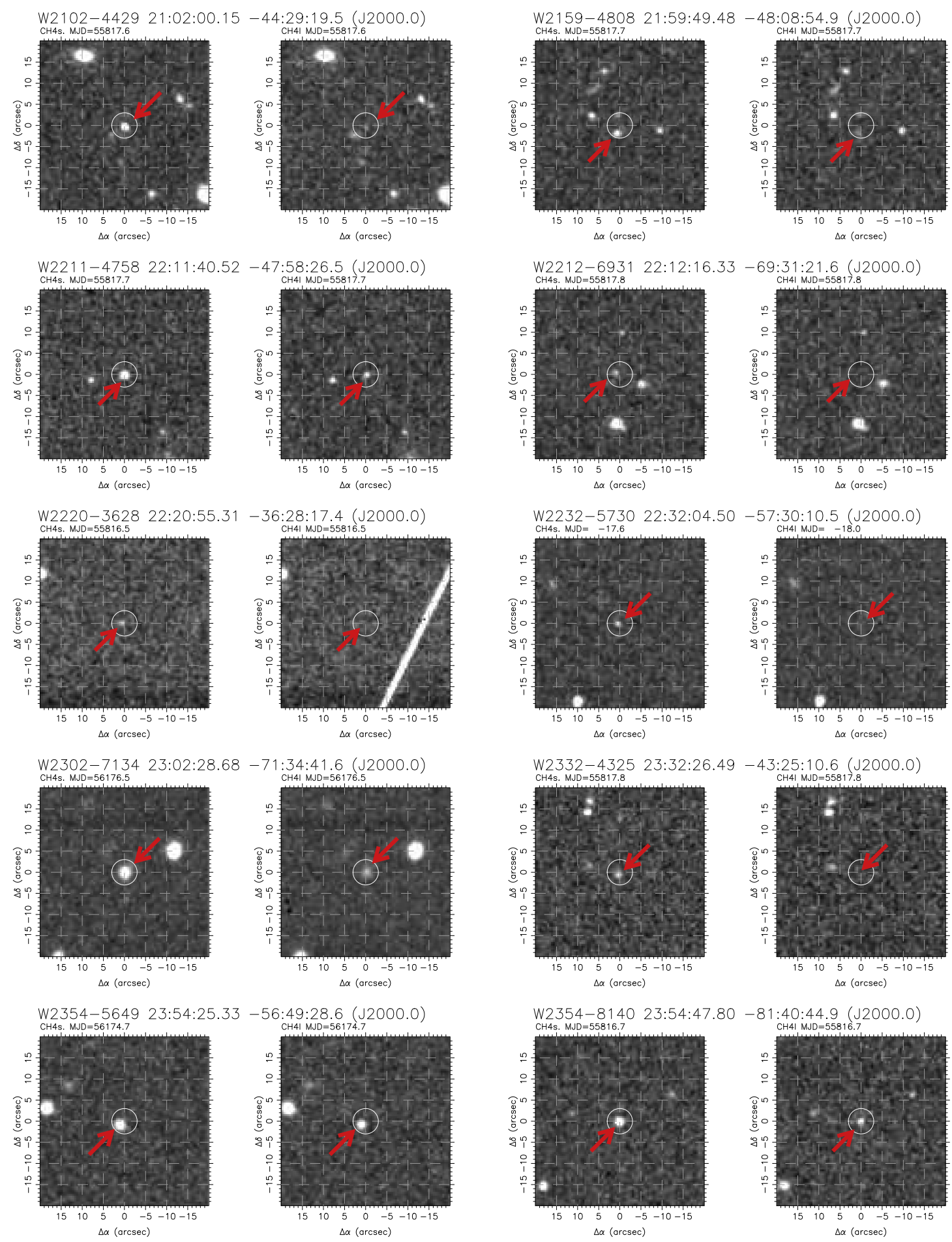

Figure 1. (Continued.)

that outlined in Tinney et al. (2012). That is, 2MASS sources in the field of view were calibrated onto the $J_{\mathrm{MKO}}$ system, and we used this MKO photometry for stars in the range $0.4<(\mathrm{J}-\mathrm{K})_{\mathrm{MKO}}<0.8$ to define the zero-point for the $\mathrm{J} 2$ and J3 magnitude system. Table 4 presents $\mathrm{J} 2$ and $\mathrm{J} 3$ photometry from Magellan, as well as WISE W1 and W2 photometry (as contained in the 2012 March WISE All-Sky data release).

\section{Imaging Summary}

The WISE candidates observed naturally fall into a few classes:

(a) A bright (i.e., $J<19.5$ ) object is readily detected as a positional match with the WISE source. In $100 \%$ of cases, these objects are subsequently confirmed by methane imaging as $\mathrm{T}$ dwarfs. 

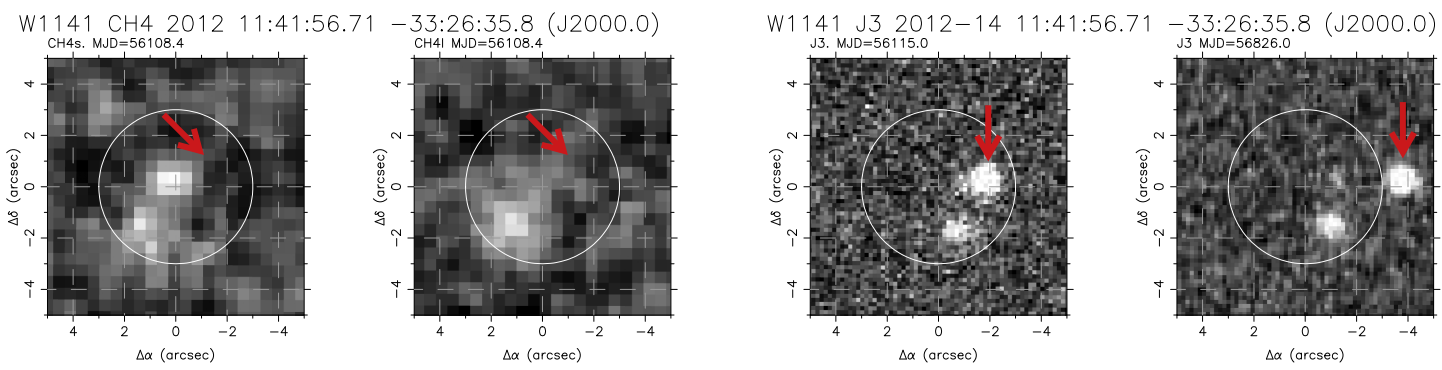

Figure 2. Zoomed-in images $10^{\prime \prime}$ on a side at the location of W1141-3326. $\mathrm{CH}_{4} \mathrm{~s}$ and $\mathrm{CH}_{4} 1$ images from 2012 are shown on the left, and J3 images from 2012 and 2014 are shown on the right. W1141-3326's proper motion moved it $2^{\prime \prime}$ to the W between 2012 July and 2014 June, and away from a group of background sources that clearly contaminated the $\mathrm{CH}_{4} \mathrm{l}$ photometry in 2012 , producing a spuriously red $\mathrm{CH}_{4} \mathrm{~s}-\mathrm{CH}_{4} \mathrm{l}$ color .

Table 5

Apparent $\left(\mu^{\prime}\right)$ and Proper $(\mu)$ Motions for New Cool Brown Dwarfs from WISE

\begin{tabular}{|c|c|c|c|c|c|c|}
\hline \multirow{2}{*}{ Object $^{\mathrm{a}}$} & \multicolumn{3}{|c|}{ WISE -IRIS2/FouStar } & \multicolumn{3}{|c|}{ FourStar alone } \\
\hline & $\mu_{\alpha}^{\prime}$ & $\begin{array}{c}\mu_{\delta}^{\prime} \\
\left(\operatorname{mas~yr}^{-1}\right)\end{array}$ & Unc. & $\mu_{\alpha}$ & $\begin{array}{c}\mu_{\delta} \\
\left(\operatorname{mas~yr}^{-1}\right)\end{array}$ & Unc. \\
\hline W0015-4615 & +720 & -450 & \pm 250 & & & \\
\hline W0032-4946 & -714 & -1300 & \pm 250 & & & \\
\hline W0042-5840 & +122 & -140 & \pm 120 & & & \\
\hline W0241-3653 & +350 & +100 & \pm 230 & & & \\
\hline W0302-5817 & -86 & -150 & \pm 120 & & & \\
\hline W0309-5016 & +645 & +100 & \pm 270 & & & \\
\hline W0404-6420 & -139 & +180 & \pm 155 & & & \\
\hline W0628-8057 & +110 & -550 & \pm 150 & & & \\
\hline W0645-0302 & -43 & -430 & \pm 160 & & & \\
\hline W0914-3459 & -160 & -20 & \pm 180 & & & \\
\hline W0940-2208 & -70 & +30 & \pm 160 & & & \\
\hline W1023-3151 & -220 & +250 & \pm 110 & & & \\
\hline W1055-1652 & -728 & +250 & \pm 160 & & & \\
\hline W1112-3857 & +510 & +590 & \pm 180 & +666 & +638 & \pm 10 \\
\hline W1141-3326 & -915 & +120 & \pm 180 & -897 & -84 & \pm 6 \\
\hline W1221-3136 & +550 & +360 & \pm 180 & & & \\
\hline W1433-0837 & -130 & 0 & \pm 240 & & & \\
\hline W1448-2534 & +110 & -670 & \pm 180 & & & \\
\hline W1501-4004 & +580 & -290 & \pm 170 & & & \\
\hline W1729-7530 & +30 & -230 & \pm 120 & & & \\
\hline W1735-8209 & -350 & -540 & \pm 230 & & & \\
\hline W2017-3421 & +385 & +10 & \pm 240 & & & \\
\hline W2049-4431 & +415 & -585 & \pm 120 & & & \\
\hline W2102-4429 & -250 & -340 & \pm 240 & +41 & -354 & \pm 3 \\
\hline W2159-4808 & +275 & -1380 & \pm 240 & & & \\
\hline W2211-4758 & -170 & -160 & \pm 240 & & & \\
\hline W2212-6931 & +600 & +200 & \pm 240 & & & \\
\hline W2220-3628 & +320 & +60 & \pm 250 & +283 & -94 & \pm 4 \\
\hline W2232-5730 & +240 & +80 & \pm 170 & & & \\
\hline W2302-7134 & -220 & 0 & \pm 160 & & & \\
\hline W2332-4325 & +200 & -315 & \pm 250 & +248 & -256 & \pm 4 \\
\hline W2354-5649 & +390 & -330 & \pm 160 & & & \\
\hline W2354-8140 & +30 & -15 & \pm 230 & & & \\
\hline
\end{tabular}

Note.

${ }^{\mathrm{a}}$ WISE designations follow Table 2.

(b) A fainter (i.e., $19.5<J<21.5$ ) object is detected as a positional match that (in some cases) we have been able to verify is a T or Y dwarf using either methane imaging, or spectroscopy obtained on other telescopes in parallel with this methane imaging program).

(c) Either a fainter (i.e., $19.5<J<21.5$ ) object is detected as a positional match with photometry that is inconsistent with this object being a T/Y dwarf, or we observe the
Table 6

WISE Cool Dwarf Spectroscopy

\begin{tabular}{|c|c|c|c|c|}
\hline WISE Designation $^{\mathrm{a}}$ & UT Date & Telescope & $\begin{array}{l}\text { Exp. } \\
\text { (s) }\end{array}$ & $\begin{array}{l}\text { Sp. } \\
\text { Type }\end{array}$ \\
\hline J001505.87-461517.6 & 2012 Jan 05 & Magellan $^{\mathrm{b}}$ & 1014 & $\mathrm{~T} 8$ \\
\hline J003231.09-494651.4 & 2012 Jan 05 & Magellan $^{\mathrm{b}}$ & 1268 & T8.5 \\
\hline J024124.73-365328.0 & 2011 Sep 08 & $\mathrm{Keck}^{\mathrm{b}}$ & 600 & $\mathrm{~T} 7$ \\
\hline AJ030237.53-581740.3 & 2017 Jan 05 & Magellan ${ }^{\mathrm{c}}$ & 2700 & Y0: \\
\hline J064528.38-030248.2 & 2013 Mar 04 & IRTF & 2400 & T6 \\
\hline J071301.84-585445.1 & 2012 May 06 & Magellan $^{\mathrm{b}}$ & 1200 & T9 \\
\hline J091408.96-345941.5 & 2016 Mar 23 & Magellan $^{\mathrm{c}}$ & 900 & $\mathrm{~T} 8$ \\
\hline J094020.10-220820.5 & 2013 Mar 04 & IRTF & 1600 & $\mathrm{~T} 8$ \\
\hline AJ102313.22-315126.7 & 2016 Mar 23 & Magellan $^{\mathrm{c}}$ & 900 & $\mathrm{~T} 8$ \\
\hline J105553.59-165216.3 & 2013 Mar 22 & Magellan $^{\mathrm{c}}$ & 800 & T9: \\
\hline J111239.24-385700.7 & 2012 May 06 & Magellan $^{\mathrm{b}}$ & 1680 & T9 \\
\hline J114156.71-332635.8 & 2014 Mar 10 & Magellan $^{\mathrm{c}}$ & 2000 & Y0 \\
\hline J143311.42-083736.4 & 2012 Jan 17 & Magellan $^{\mathrm{b}}$ & 240 & $\mathrm{~T} 8$ \\
\hline J144806.48-253420.3 & 2014 Mar 10 & Magellan $^{\mathrm{c}}$ & 800 & $\mathrm{~T} 8$ \\
\hline $\mathrm{J} 150115.92-400418.4$ & 2014 Mar 04 & IRTF & 2000 & T6 \\
\hline AJ172907.10-753017.0 & 2016 Mar 23 & Magellan $^{\mathrm{c}}$ & 900 & $\mathrm{~T} 7$ \\
\hline J173551.72-820900.1 & 2016 Mar 23 & Magellan $^{\mathrm{c}}$ & 600 & T6 \\
\hline $\mathrm{J} 210200.15-442919.5$ & 2011 Oct 09 & $\mathrm{Keck}^{\mathrm{d}}$ & 4200 & T9 \\
\hline J215949.48-480854.9 & 2012 May 05 & Magellan $^{\mathrm{b}}$ & 1400 & T9 \\
\hline J221216.33-693121.6 & 2012 May 06 & Magellan $^{\mathrm{b}}$ & 1400 & T9.5 \\
\hline $\mathrm{J} 223204.50-573010.5$ & 2012 May 06 & Magellan $^{\mathrm{b}}$ & 1400 & T9 \\
\hline J233226.49-432510.6 & 2011 Oct 09 & $\mathrm{Keck}^{\mathrm{d}}$ & 4200 & T9: \\
\hline J235425.33-564928.6 & 2016 Nov 18 & Magellan $^{\mathrm{c}}$ & 1200 & T6 \\
\hline
\end{tabular}

Notes.

${ }^{\text {a }}$ WISE designations follow Table 2 .

${ }^{\mathrm{b}}$ Data processing carried out with FIREHOSE package, as described in the text.

${ }^{c}$ Data processing carried out with Figaro package, as described in the text.

${ }^{d}$ These are the same spectra used to obtain spectral types by Kirkpatrick et al. (2012), albeit independently classified in this paper (Figure 3). In both cases we obtain the same types as Kirkpatrick et al.

Table 7

Adopted Spectral Standards

\begin{tabular}{lll}
\hline \hline $\begin{array}{l}\text { Spectral } \\
\text { Type }\end{array}$ & \multicolumn{1}{c}{$\begin{array}{c}\text { Full } \\
\text { Designation }\end{array}$} & $\begin{array}{l}\text { Short } \\
\text { Designation }\end{array}$ \\
\hline T5 & 2MASS J15031961+2525196 & 2M1503 \\
T6 & SDSS J162414.37+002915.6 & S1624 \\
T7 & 2MASS J07271824+1710012 & 2M0727 \\
T8 & 2MASS J04151954-0935066 & 2M0415 \\
T9 & UGPS J072227.51-054031.2 & U0722 \\
T9.5 & WISE J014807.25-720258.7 & W0148 \\
Y0 & WISEPA J173835.53+273258.9 & W1738 \\
Y0.5 & WISEPA J154151.66-225025.2 & W1541 \\
Y1 & WISE J035000.32-565830.2 & W0350 \\
\hline
\end{tabular}



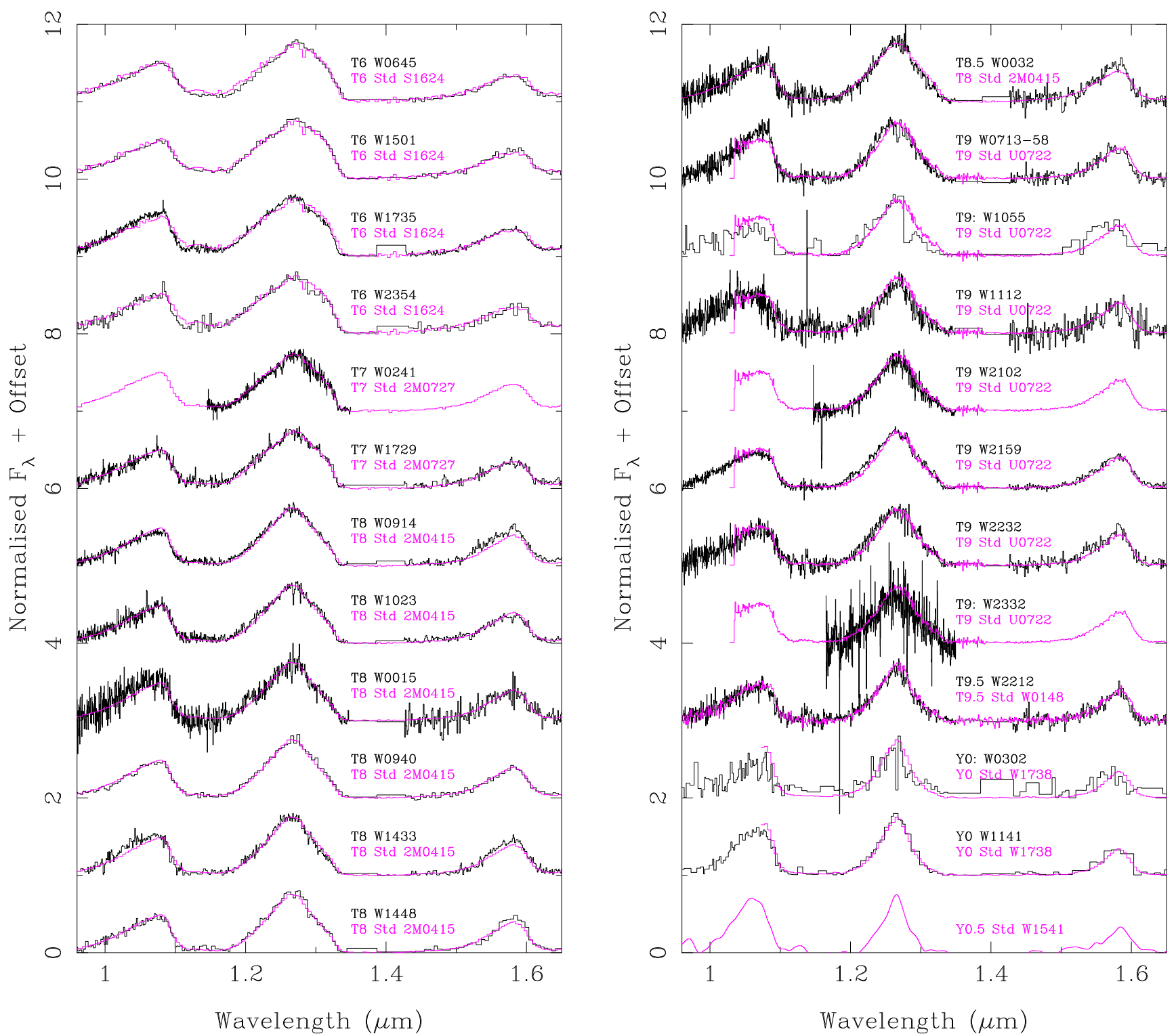

Figure 3. $\mathrm{T}$ and $\mathrm{Y}$ dwarf spectra listed in Table 6 plotted to demonstrate spectral classifications derived as described in the text. Each new spectrum (black lines) is overplotted with the relevant spectral standard (magenta lines).

object to be resolved in good imaging at Magellan, indicating the WISE source is most likely a galaxy.

(d) No counterpart is detected down to $\mathrm{J} \sim 21.5-22-$ these could either be contaminant sources (i.e., non-cool-brown dwarfs whose WISE photometry mimics those of brown dwarfs due to confusion, photometric scatter, etc.) or cool brown dwarfs fainter than $J \sim 22$.

Finding charts for objects newly identified as being $\mathrm{T}$ or $\mathrm{Y}$ dwarfs are provided in Figure 1. Figure 2 shows expanded images at the position of the new Y0 dwarf W1141-3326. These show how the $2012 \mathrm{CH}_{4} \mathrm{~s}-\mathrm{CH}_{4} 1$ photometry for this object was contaminated by confusion with a background source. The images from 2014 (by which time W1141-2236 had moved $\approx 2^{\prime \prime}$ to the west) show W1141-2236 clearly separated from this background source. It is also clear that in the 2012 images, $\mathrm{CH}_{4} \mathrm{~s}$ is more contaminated than $\mathrm{CH}_{4}$ l, making $\mathrm{CH}_{4} \mathrm{~S}-\mathrm{CH}_{4} \mathrm{l}$ more positive than it should be. We therefore exclude W1141-3336 from our updated $\mathrm{CH}_{4} \mathrm{~S}$ $-\mathrm{CH}_{4}$ l-to-spectral-type calibration in Section 6.1.

\subsection{Brown Dwarf Proper Motions}

Figure 1 highlights the complicating factor of proper motion when seeking to identify cool brown dwarfs. The charts for W2049-4431, W2159-4808, W1112-3857, and W1141-3326 (for example) all show substantial motion between the epoch of the WISE detection (mean WISE epochs are in the range 2010 April-June for these four targets) and the epoch of follow-up (from 2011 September to 2017 January).

The observed motions of our new WISE $\mathrm{T}$ and $\mathrm{Y}$ dwarfs are summarized in Table 5. We obtained astrometry for our J, $\mathrm{CH}_{4} \mathrm{~s}$, or J3 images by cross-matching against the 2MASS PSC and relying on WISE and the PSC being on the same coordinate system. The individual positional uncertainty of the WISE objects $(0$ "' $3-0$ "' 5$)$ is the dominant source of uncertainty, so this 

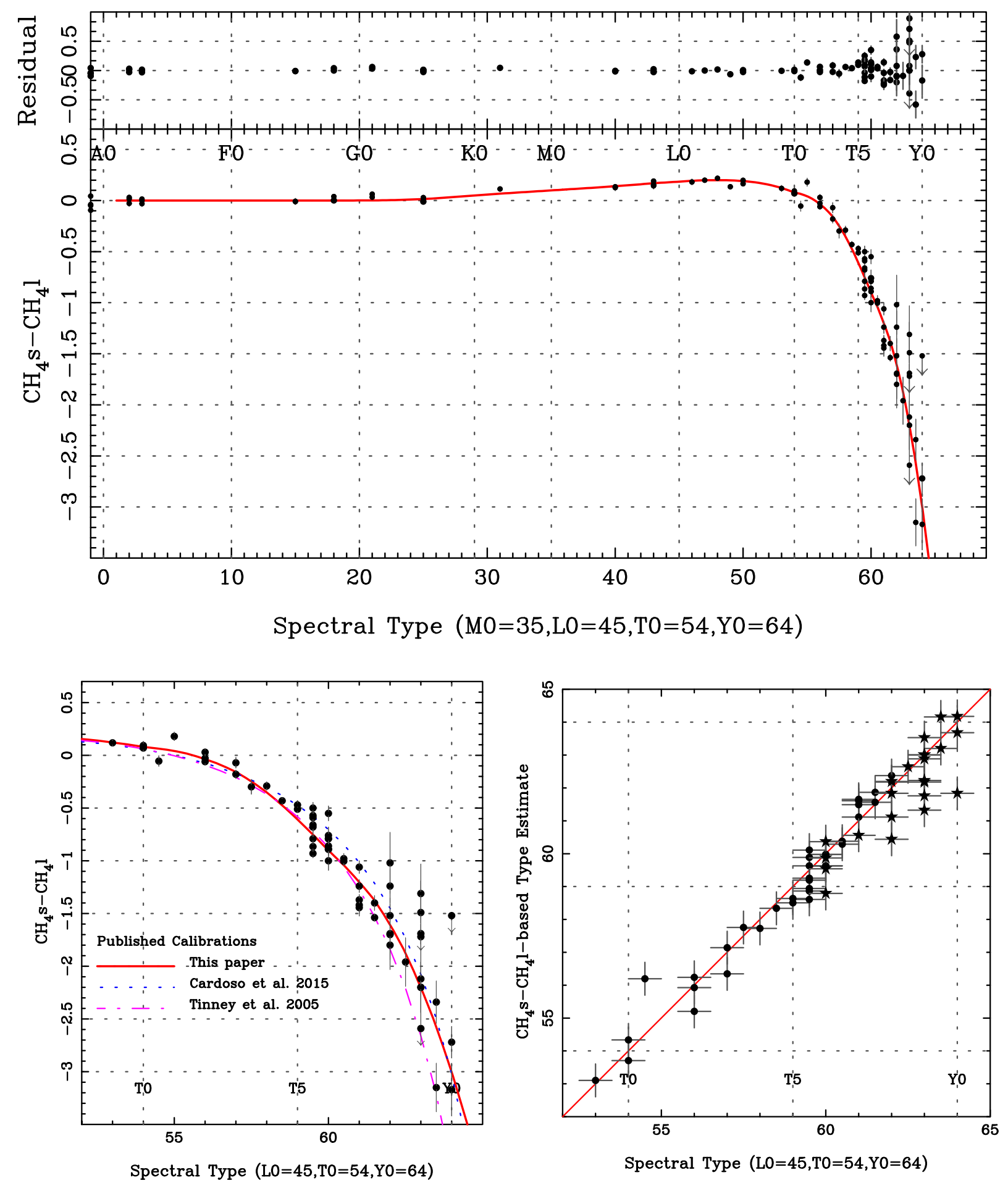

Figure 4. Upper panel: methane-sensitive $\mathrm{CH}_{4} \mathrm{~s}-\mathrm{CH}_{4} \mathrm{l}$ as a function of A-Y spectral type. The uncertainties plotted are a combination of photon-counting uncertainties, aperture correction uncertainties, and photometric calibration uncertainties. Typical uncertainties on spectral types (not plotted) are \pm 0.5 . The plotted parameterization is a spline constrained at the locations in Table 8. rms scatter about the parameterization is 0.18 mag for the whole range, 0.06 mag for L0-T2 dwarfs, and $0.12 \mathrm{mag}$ for T3-T8 dwarfs, and $0.45 \mathrm{mag}$ for T9-Y0 dwarfs. The corresponding spectral type scatters are 0.6 sub-type for L0-T2 dwarfs and 0.43 sub-type for T3T8 dwarfs, and 0.6 sub-type for T9-Y0 dwarfs. Lower right panel: zoomed-in view of the T and Y dwarf regions as seen in the upper panel, with the previously published calibration relations due to Tinney et al. (2005) and Cardoso et al. (2015) also shown. Lower left panel: comparison of the predicted spectral types from this $\mathrm{CH}_{4} \mathrm{~s}-\mathrm{CH}_{4} 1$ relation with observed spectral types (solid circles are from Tinney et al. (2005); solid stars are from this paper). 

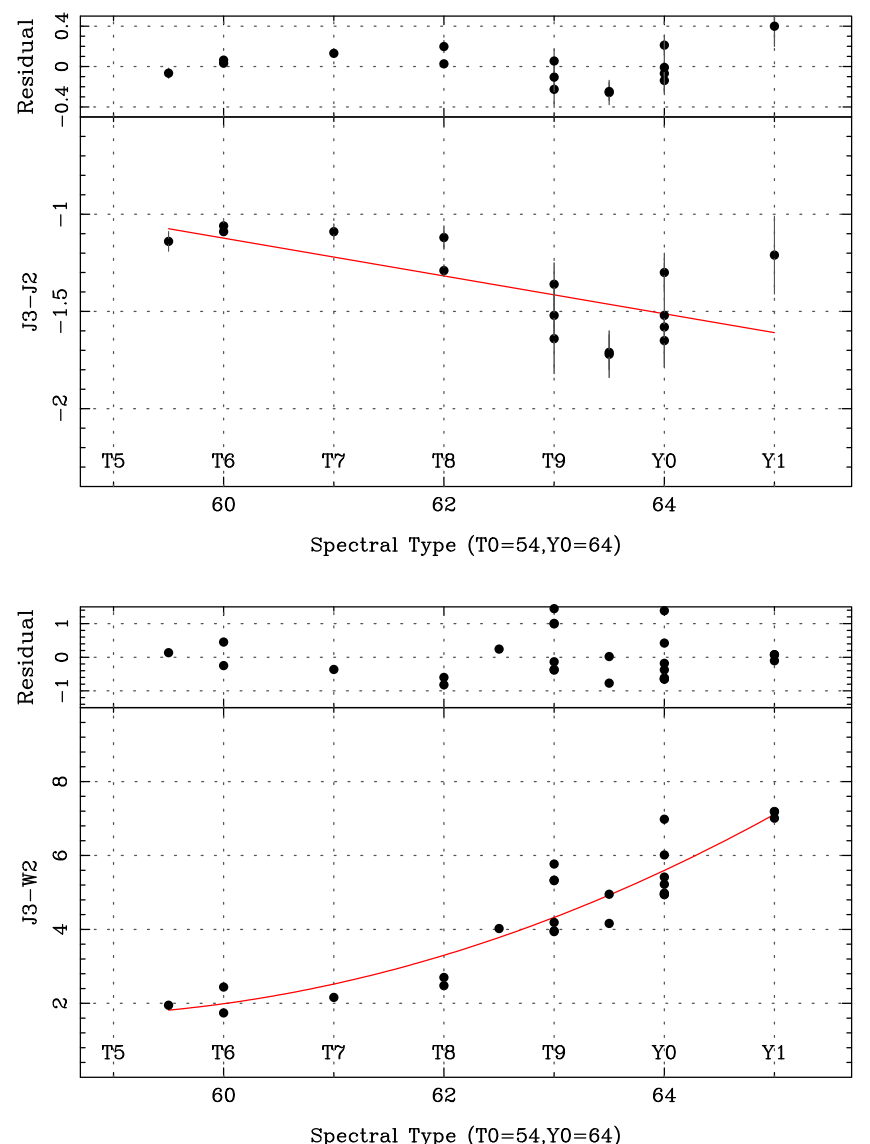

Figure 5. (a) Upper panel: methane-sensitive J3-J2 as a function of T5.5-Y1 spectral type. The uncertainties plotted are a combination of photon-counting uncertainties, aperture correction uncertainties, and photometric calibration uncertainties. Typical uncertainties on spectral types (not plotted) are \pm 0.5 . The plotted linear fit is described in Section 6.2, and has an rms of 0.19 mag. (b) Lower panel: temperature-sensitive J3-W2 as a function of T5.5-Y1 spectral type. Along with the objects in Table 4, we also show nine objects with J3 and W2 (but without J2) from Tinney et al. (2014). The uncertainties are the same as the upper panel. The plotted parameterization is a quadratic fit described in Section 6.3, with an rms of 0.65 mag.

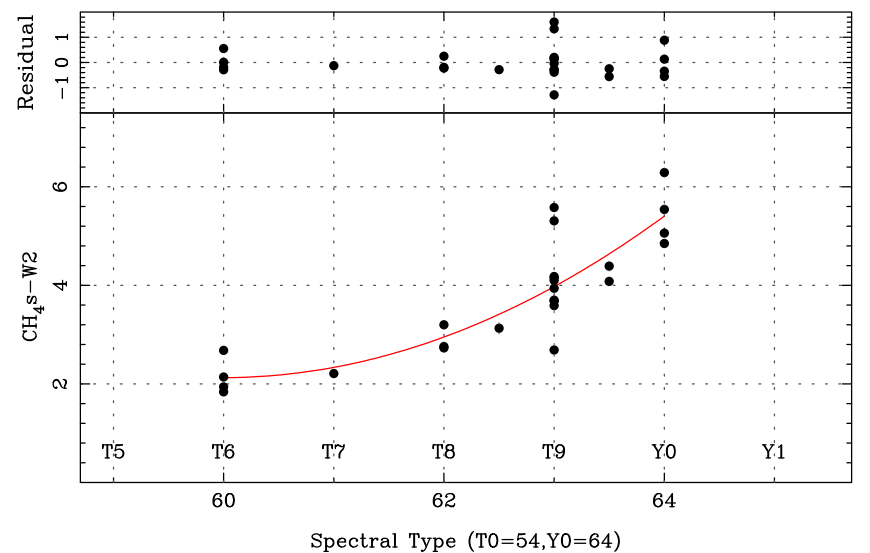

Figure 6. Temperature-sensitive $\mathrm{CH}_{4} \mathrm{~s}-\mathrm{W} 2$ as a function of T6-Y0 spectral type. Uncertainties are the same as those in Figure 5. The plotted parameterization is the quadratic fit described in Section 6.3, with an rms of $0.60 \mathrm{mag}$.

is a good assumption. Note that we distinguish between the "apparent" motion of our targets from just two epochs as observed by WISE and our IRIS2 or FourStar follow-up
Table 8

$\mathrm{CH}_{4} \mathrm{~S}-\mathrm{CH}_{4} \mathrm{l}$ vs. Spectral Type

\begin{tabular}{lccccc}
\hline \hline SpT & $\mathrm{CH}_{4} \mathrm{~s}-\mathrm{CH}_{4} \mathrm{l}$ & $\mathrm{SpT}$ & $\mathrm{CH}_{4} \mathrm{~s}-\mathrm{CH}_{4} \mathrm{l}$ & $\mathrm{SpT}$ & $\mathrm{CH}_{4} \mathrm{~s}-\mathrm{CH}_{4} 1$ \\
\hline A0 & 0.000 & $\mathrm{M} 4$ & 0.132 & $\mathrm{~T} 2$ & -0.035 \\
$\mathrm{~A} 5$ & 0.000 & $\mathrm{M} 6$ & 0.150 & $\mathrm{~T} 3$ & -0.157 \\
$\mathrm{~F} 0$ & 0.000 & $\mathrm{M} 8$ & 0.169 & $\mathrm{~T} 4$ & -0.350 \\
F5 & 0.000 & $\mathrm{~L} 0$ & 0.185 & $\mathrm{~T} 5$ & -0.595 \\
$\mathrm{G} 0$ & 0.001 & $\mathrm{~L} 2$ & 0.199 & $\mathrm{~T} 6$ & -0.900 \\
$\mathrm{G} 5$ & 0.015 & $\mathrm{~L} 4$ & 0.198 & $\mathrm{~T} 7$ & -1.250 \\
$\mathrm{~K} 0$ & 0.050 & $\mathrm{~L} 6$ & 0.178 & $\mathrm{~T} 8$ & -1.600 \\
$\mathrm{~K} 3$ & 0.076 & $\mathrm{~L} 8$ & 0.123 & $\mathrm{~T} 9$ & -2.200 \\
M0 & 0.099 & $\mathrm{~T} 0$ & 0.080 & $\mathrm{Y} 0$ & -3.000 \\
M2 & 0.115 & $\mathrm{~T} 1$ & 0.043 & $\mathrm{Y} 1$ & -4.000 \\
\hline
\end{tabular}

imaging (here denoted $\mu^{\prime}$ ), and the true proper motion from an astrometric solution that includes parallax. The large WISE position uncertainties mean that the uncertainty in the right ascension and declination components of $\mu^{\prime}$ are very similar, so only a single uncertainty is quoted for both in the table. This uncertainty includes an additional term added in quadrature of 100 mas to account for possible parallax motion not being accounted for in objects that could be as close as $10 \mathrm{pc}$ away. Five of these brown dwarfs are the subject of our ongoing astrometric program with FourStar, so we provide preliminary proper motion solutions (in this case including a parallax solution) for comparison. In each case the solutions agree, although the FourStar astrometric solutions are between 10 and 20 times better than the WISE-IRIS2/FourStar estimates.

Motions of $0.15 \mathrm{yr}^{-1}$ are not uncommon, and the motions observed for the most rapidly moving brown dwarfs (W0032-

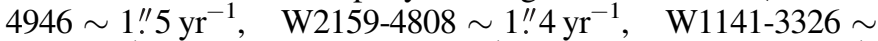
0 ". $95 \mathrm{yr}^{-1}, \mathrm{~W} 1112-3857 \sim 0$ ". $9 \mathrm{yr}^{-1}, \mathrm{~W} 2049-4431 \sim 0$ ". $7 \mathrm{yr}^{-1}$ ) highlight how identification via "blind" spectroscopy becomes substantially more difficult after just a few years. After 5 years the positional error box that must be searched can be as large as $20^{\prime \prime}$ on a side, and can contain multiple objects that would have to be spectroscopically observed in turn to find the correct $\mathrm{T} / \mathrm{Y}$ dwarf. In this situation methane imaging becomes an even more powerful tool to pick out the correct counterpart.

\section{Observations-Spectroscopy}

Follow-up spectroscopy is the "gold standard" for confirming a $\mathrm{T}$ or Y dwarf identification. Spectroscopic observations have therefore been carried out by members of the WISE Science Team brown dwarf collaboration on a variety of telescopes in parallel with our imaging program at the AAT and Magellan. We report here spectroscopy obtained on multiple nights between 2011 September 8 and 2017 January 05 (Table 6).

\subsection{Magellan/FIRE}

The Folded-port Infrared Echellette (FIRE; Simcoe et al. 2008, 2010) at the $6.5 \mathrm{~m}$ Walter Baade Telescope on Cerro Manqui at the Las Campanas Observatory, Chile, uses a $2048 \times 2048$ HAWAII-2RG array. In prism mode, it covers a wavelength range from 0.8 to $2.5 \mu \mathrm{m}$ at a resolution ranging from $R=500$ at the J-band to $R=300$ at the K-band for a slit width of 0 ." 6 . FIRE was used to obtain spectroscopy of T dwarf candidates on the nights listed in Table 6. FIRE reductions in 
Table 9

$\mathrm{J} 3-\mathrm{W} 2, \mathrm{CH}_{4} \mathrm{~s}-\mathrm{W} 2$ vs. Spectral Type

\begin{tabular}{lcccccccc}
\hline \hline SpT & J3-W2 & J3-W2 & SpT & J3-W2 & J3-W2 & SpT & J3-W2 & J3-W2 \\
\hline T5 & 1.70 & $\cdots$ & T8 & 3.30 & 2.95 & Y0 & 5.60 & 5.41 \\
T6 & 1.98 & 2.13 & T9 & 4.32 & 3.97 & Y0.5 & Y & 7.11 \\
T7 & 2.52 & 2.34 & T9.5 & 4.93 & 4.64 & Y1 & $\ldots$ \\
\hline
\end{tabular}

Table 10

Comparison with Extant Spectroscopy

\begin{tabular}{lcccl}
\hline \hline Object & $\mathrm{CH}_{4} \mathrm{~s}-\mathrm{CH}_{4} 1$ “Type" & SpT (this paper) & SpT (other) & Reference \\
\hline W0404-6420 & $\mathrm{T} 7.1 \pm 0.5$ & $\ldots$ & $\mathrm{T} 9$ & Schneider et al. (2015) \\
W1055-1652 & $>\mathrm{T} 8.2$ & $\mathrm{~T} 9 \pm 0.5$ & $\mathrm{~T} 9.5$ & E. C. Martin et al. (2018, in preparation) \\
W1433-0837 & $\ldots$ & $\mathrm{T} 8 \pm 0.5$ & $\mathrm{~T} 8$ & Lodieu et al. (2012) \\
W1448-2534 & $\mathrm{T} 6.4 \pm 0.5$ & $\mathrm{~T} 8 \pm 0.5$ & $\mathrm{~T} 8$ & Thompson et al. (2013) \\
W2212-6931 & $\mathrm{Y} 0.2 \pm 0.5$ & $\mathrm{~T} 9.5 \pm 0.5$ & $\mathrm{~T} 9$ & Schneider et al. (2015) \\
\hline
\end{tabular}

this paper were carried out in two ways. Roughly half the spectra were processed in the same manner as the spectroscopy presented in Tinney et al. (2012) using the Figaro data reduction package, with telluric removal carried out using A0 spectra acquired immediately before or after the science target at similar airmass. Remaining objects were processed using the FIREHOSE package for low-dispersion data following the procedure in the online "cookbook."14 For our faintest objects, pair subtraction prior to insertion into the pipeline greatly improved the accuracy of the sky-line fitting procedure. The combined spectrum was then corrected for telluric absorption and flux-calibrated using observations of an A0 V star and the technique described in Vacca et al. (2003) and the XTELLCOR program from SpeXtool (see Cushing et al. 2004).

\subsection{Keck/NIRSPEC}

The Near-Infrared Spectrometer (NIRSPEC; McLean et al. 1998, 2000) at the $10 \mathrm{~m} \mathrm{W.} \mathrm{M.} \mathrm{Keck} \mathrm{Observatory} \mathrm{on}$ Maunakea, Hawai'i, was used to obtain confirmation spectroscopy of several new T dwarfs. For spectroscopy, NIRSPEC uses a $1024 \times 1024 \mathrm{InSb}$ array. The NIRSPEC observations employed the $42^{\prime \prime} \times 0$ " 57 slit, providing a resolution $R \sim$ 1500. Our brown dwarf candidates were observed in the N3 configuration (see McLean et al. 2003) that covers part of the $\mathrm{J}$-band window from 1.15 to $1.35 \mu \mathrm{m}$. Data reduction made use of the publicly available REDSPEC package, with modifications to remove residuals from the sky-subtracted pairs prior to 1D spectral extraction.

\subsection{IRTF/SpeX}

SpeX is a medium-resolution spectrograph and imager at NASA's 3 m Infrared Telescope Facility (IRTF) on Maunakea, Hawai'i. It uses a $1024 \times 1024 \mathrm{InSb}$ array for its spectroscopic observations (Rayner et al. 2003). We used the prism mode with a 0 . 5 wide slit to achieve a resolving power of $R \equiv$ $\lambda / \Delta \lambda \approx 150$ over the range $0.8-2.5 \mu \mathrm{m}$. A series of $200 \mathrm{~s}$ exposures were typically obtained at two different positions along the $15^{\prime \prime}$ long slit. A0 dwarf stars were observed soon after or before the target and at similar airmass, and used for telluric correction and flux calibration. A set of exposures of internal

\footnotetext{
${ }^{14}$ See http://www.mit.edu/people/rsimcoe/FIRE/ob_data.htm for details.
}

flat-field and argon arc lamps were obtained for flat-fielding and wavelength calibration.

The data were reduced using Spextool (Cushing et al. 2004), the IDL-based data reduction package for SpeX. The raw images were first corrected for nonlinearity, pair-subtracted, and then flat-fielded. For some of the fainter sources, multiple pair-subtracted images were averaged in order to facilitate tracing. The spectra were then optimally extracted (e.g., Horne 1986) and wavelength-calibrated using the argon lamp exposures. Multiple spectra were then averaged and the resulting spectrum was corrected for telluric absorption and flux-calibrated using observations of an A0 V star using the technique described in Vacca et al. (2003).

\subsection{Spectral Types}

The objects listed in Table 6 were spectrally classified using the near-infrared T0-to-T8 dwarf sequence of Burgasser et al. (2006), extended to later $\mathrm{T}$ and $\mathrm{Y}$ dwarfs by Cushing et al. (2011) and Kirkpatrick et al. (2012). (See Table 7 for the specifically adopted standard spectra.) Assignment of types was performed by overplotting these standards onto the candidate spectra and determining by eye which standard provided the best match. In some cases two adjacent standards, such as T8 and T9, provided an equally good match, so the candidate spectrum was assigned an intermediate type (in this example, T8.5). In Figure 3 we show the near-infrared spectra for all of our sources compared with the relevant spectral standard. We consider these types to have an uncertainty of \pm 0.5 sub-types. Types that are more uncertain due to low signal-to-noise ratio spectra (i.e., \pm 1 sub-type) are marked with a colon ":" next to the spectral type.

\section{Discussion}

\subsection{An Updated $\mathrm{CH}_{4} \mathrm{~s}-\mathrm{CH}_{4}$ l Spectral Type Calibration}

Tinney et al. (2005) presented a calibration between the IRIS2 $\mathrm{CH}_{4} \mathrm{~s}-\mathrm{CH}_{4} \mathrm{l}$ color and spectral type. Cardoso et al. (2015) used very similar filters on the NICS instrument on the Telescope Nazionale Galileo and derived a calibration using the same functional form as Tinney et al. (2005), but with slightly different values. With the additional methane imaging data for later-type objects available in Table 2, we have updated this calibration to derive the relation shown in the 
upper panel of Figure 4. A relation with a simple functional form (like that used by Tinney et al.) proved to be impossible to obtain, so we have instead used a spline calibration, which we present as the sequence shown in Table 8. The scatter about this calibration over the whole spectral type range is $0.11 \mathrm{mag}$. More importantly, for the use of $\mathrm{CH}_{4} \mathrm{~s}-\mathrm{CH}_{4} \mathrm{l}$ colors to estimate spectral types, the slope of the relation for types beyond T5 is very steep, so that typical measurement errors of $\pm 0.1 \mathrm{mag}$ in the color map turn into uncertainties in spectral type estimates of less than \pm 0.3 sub-types for all objects later than $T 5$. We therefore adopt an uncertainty, for our estimated $\mathrm{T}$ and $\mathrm{Y}$ types in Table 2, of better than 0.5 sub-types.

The lower left panel of Figure 4 shows an expanded version of this plot, along with the calibration relations from Tinney et al. (2005) and Cardoso et al. (2015). The authors of the latter have already noted that their calibration is systematically redder (in $\mathrm{CH}_{4} \mathrm{~s}-\mathrm{CH}_{4} \mathrm{l}$ ) for a given spectral type than the earlier calibration. Examination of their Figure 4 suggests that this may be due to either a fortuitous observation of a sample of objects lying above the sequence of Tinney et al. (2005), or a systematic difference in the color terms driven by the optics of NICS on the Telescopio Nazionale Galileo, or both. (The filters and detectors in both IRIS2 and NICS are identical, so only the rest of the optical train can plausibly produce this difference.) The addition of further very late objects in our new sample of $\mathrm{T}$ and $\mathrm{Y}$ dwarfs brings our new calibration and that of Cardoso et al. (2015) into much closer alignment for all objects later than T7, while the differences at earlier types now seem consistent with cosmic scatter about the calibration.

The lower right panel of Figure 4 plots the resulting estimated spectral types against actual spectral types, for objects from both this paper and Tinney et al. (2005). In the vast majority of cases, the estimated spectral types predicted based on $\mathrm{CH}_{4} \mathrm{~S}-\mathrm{CH}_{4} \mathrm{l}$ color are in line with the spectroscopic types $-70 \%$ of the objects plotted have estimated types agreeing with their observed types to within \pm 0.5 types, and $91 \%$ agree to within \pm 1 type. The most prominent exception is W1141-3326, which (as already noted) was found to be much later when a spectrum was acquired (Y0) than predicted (T5), due to confusion with a background object. Of the five objects observed to deviate by more than one whole spectral type from their predicted type, four are deviant in the sense that the observed type was later than predicted. Indeed, only 1 of 53 objects was found to be earlier than predicted by more than a whole spectral type, which reinforces the power of these filters for robustly and rapidly identifying (and approximately typing) T- and Y-type dwarfs - if imaging detects a methane absorption signature in an unresolved object (i.e., not a galaxy) near the position of a WISE or 2MASS candidate object, then it is almost certainly a legitimate match with the WISE or 2MASS candidate, making it a T or Y dwarf. If the estimated type that results is in "error" it is more likely to predict the object to be earlier, rather than later. (A similar result was found by Cardoso et al. 2015.) These results give us confidence in the $\mathrm{T}$ dwarf identifications, even for the eight $\mathrm{T}$ dwarfs in Tables 2 and 4 for which spectroscopy has not yet been obtained.

\subsection{J3-J2 Versus Spectral Type}

To observe fainter cool dwarfs (i.e., $J \gtrsim 21$ candidates where methane observations in the $\mathrm{H}$-band with $\mathrm{CH}_{4} \mathrm{~s}, \mathrm{CH}_{4} \mathrm{l}$ filters on the $4 \mathrm{~m}$ AAT became problematic) we made use of methane-sensitive filters in the J-band (i.e., the J3 and J2 filters installed in FourStar). These J-band filters see a substantially lower sky background than the equivalent H-band filters, while the blue colors of late T and Y dwarfs mean they see essentially the same flux as in the H-band. This combination makes it feasible to target fainter objects down to $J \sim 22-24-$ and in one extreme case down to $\mathrm{J} 3 \sim 25$ (Faherty et al. 2014).

We take this opportunity to note that the faintest groundbased near-infrared detection of a Y dwarf (WISE J085510.83071442.5 at $\mathrm{J} 3=24.8_{-0.35}^{+0.5}$ ) reported by Faherty et al. (2014) has been the subject of some debate (Luhman \& Esplin 2016; Schneider et al. 2016a). The claimed discrepancy here is almost certainly not real, but rather a result of issues associated with conversion to a standard MKO J passband from the measured bandpasses (in order of decreasing width): HST filter F125W $1.10-1.40 \mu \mathrm{m}$ for Schneider et al.; J3 $=1.21-1.37 \mu \mathrm{m}$ for Faherty et al.; and HST filter F127M 1.24-1.31 $\mu \mathrm{m}$ for Luhman $\&$ Esplin. Faherty et al. measured $\mathrm{J} 3=24.8_{-0.35}^{+0.5}$, after which Schneidet et al. reported F125W $=26.41 \pm 0.27$ and Luhman $\&$ Esplin reported a mean value of F127M $=24.45 \pm 0.1$ from three observations (all Vega-magnitudes). When one considers the different bandpasses, these magnitude differences make sense. F125W includes substantial water vapor and methane absorption in its wider bandpass than J3. So the F125W Vegamagnitude should be fainter than J3. In turn J3 includes slightly more molecular absorption than F127M, which selects out a Y dwarf flux peak. The significant "discrepancy" that Schenider et al. noted with the result of Faherty et al. is not in the observed detections in these three bands, but rather in $\mathbf{J}$ magnitudes derived after conversion from the measured bandpass. For the Faherty et al. J3 detection, this was done using an empirical color correction based on (hotter) $\mathrm{T}$ and $\mathrm{Y}$ dwarfs, which is clearly not appropriate for this very cool object.

We have $\mathrm{J} 2$ and $\mathrm{J} 3$ photometry for a smaller range of spectral types than we have $\mathrm{CH}_{4} \mathrm{~s}$ and $\mathrm{CH}_{4}$ l, i.e., only spanning T5.5 to Y1. These data are plotted in the upper panel of Figure 5. Recalling that our J3-J2 system is defined so that objects in the A-G spectral type range will have $\mathrm{J} 3-\mathrm{J} 2=0$, the observation of a significantly negative $\mathrm{J} 3-\mathrm{J} 2$ color does clearly distinguish $\mathrm{T}$ and $\mathrm{Y}$ dwarfs from much hotter field stars. The simple linear fit to these data in the figure is parameterized as a function of a modified numeric spectral type $n^{\prime}$, such that $n^{\prime}=n-60$ (making $n^{\prime}=4$ equivalent to a $\mathrm{Y} 0$ ), as

$$
\mathrm{J} 3-\mathrm{J} 2=-1.12-0.097 n^{\prime},
$$

with an rms scatter of 0.19 mag.

Unfortunately, it would appear that the ability to observe fainter targets in $\mathrm{J} 3 / \mathrm{J} 2$ comes with the penalty of obtaining less information on the spectral types of those objects. The J3-J2 color appears to "saturate" at $\mathrm{J} 3-\mathrm{J} 2 \approx-1.5$ for late $\mathrm{T}$ and $\mathrm{Y}$ dwarfs-a range of spectral types over which $\mathrm{CH}_{4} \mathrm{~s}-\mathrm{CH}_{4} \mathrm{l}$ continues to become more and more negative for later objects. Moreover, Y dwarfs are only $\sim 0.5$ magnitudes more negative in J3-J2 than mid-T dwarfs, and the scatter about any trend is substantial at $\pm 0.18 \mathrm{mag}$. The equivalent numbers for $\mathrm{CH}_{4} \mathrm{~s}$ $-\mathrm{CH}_{4} \mathrm{l}$ are $\sim 3 \mathrm{mag}$ and $\pm 0.45 \mathrm{mag}$. This means that while J3-J2 can unequivocally identify a very cool brown dwarf's near-infrared counterpart (given the prior information that a cool dwarf is expected at that position from a large survey like WISE ), it cannot provide a very good estimate of how cool that brown dwarf is. 


\subsection{J3-W2 versus Spectral Type}

However, all is not lost, because the ability of $\mathrm{J} 3-\mathrm{J} 2$ to unequivocally associate a near-infrared source with a WISE thermal-infrared one means that its $\mathrm{J} 3-\mathrm{W} 2$ color is therefore determined. J3-W2 is primarily sensitive to effective temperature by sampling stellar flux in gaps between the strongest molecular absorptions over a long wavelength baseline, as has already been shown for $J-W 2$ by Cushing et al. (2011) and Kirkpatrick et al. (2012) — as distinct to the methane colors $\mathrm{CH}_{4} \mathrm{~S}-\mathrm{CH}_{4} \mathrm{l}$ and $\mathrm{J} 3-\mathrm{J} 2$, which are sensitive to effective temperature by measuring the strength of a specific molecular absorption.

As the lower panel of Figure 5 demonstrates, J3-W2 shows a pronounced trend with spectral type, and while the scatter about a quadratic fit to these data is substantial (we again parameterize this fit as a function of a modified numeric spectral type $n^{\prime}$ such that $n^{\prime}=n-60$, meaning a Y0 dwarf has $\left.n^{\prime}=4\right)$

$$
\mathrm{J} 3-\mathrm{W} 2=1.99+0.4090 n^{\prime}+0.12313 n^{\prime 2},
$$

with an $\mathrm{rms}=0.65 \mathrm{mag}$. It also spans a large range in $\mathrm{J} 3-\mathrm{W} 2$ of over $5.5 \mathrm{mag}$. This means that its discriminating power for assigning an estimated spectral type is similar to that of the $\mathrm{CH}_{4} \mathrm{~S}-\mathrm{CH}_{4} \mathrm{l}$ color over this spectral type range. For convenience, we show the value of this parameterization as a function of spectral type in Table 9.

In Figure 6 we show the equivalent plot to the lower panel of Figure 5, but based on $\mathrm{CH}_{4} \mathrm{~s}-\mathrm{W} 2$ color instead of J3-W2. The $\mathrm{CH}_{4} \mathrm{~s}-\mathrm{W} 2$ color has a similar "lever arm" on spectral type as J3$\mathrm{J} 2$, spanning a smaller range in colors (3.5 mag), with a slightly smaller rms of $0.60 \mathrm{mag}$. The equivalent polynomial fit is

$$
\mathrm{CH}_{4} \mathrm{~s}-\mathrm{W} 2=2.13+0.00519 n^{\prime}+0.20357 n^{\prime 2} .
$$

It is important to note, however, that observation of a candidate WISE counterpart in $\mathrm{J} 3$ or $\mathrm{CH}_{4} \mathrm{~S}$ (or indeed $\mathrm{J}$ or $\mathrm{H}$ ) alone is not sufficient to make a cool brown dwarf identification. $\mathrm{J} 2$ or $\mathrm{CH}_{4} \mathrm{l}$ is essential to obtain a clear identification of methane absorption. Without that identification, the association of a chance (usually faint) background source will invariably result in a large, but completely spurious, $\mathrm{J} 3-\mathrm{W} 2$ or $^{\mathrm{CH}_{4} \mathrm{~s}-\mathrm{W} 2}$ color . Both $\mathrm{J} 3$ and $\mathrm{J} 2$ methane-sensitive bands are to identify a near-infrared, methane-absorbing counterpart, following which $\mathrm{J} 3-\mathrm{W} 2$ or $\mathrm{CH}_{4} \mathrm{~s}-\mathrm{W} 2$ can provide an estimate of how cool that object is.

\subsection{New Y Dwarfs}

W0302-5817 is one of two new Y dwarfs presented for the first time in this paper. Its spectrum (obtained in 2017 January) is of low signal-to-noise but does clearly indicate a Y0 spectral type. This allows us to make a distance estimate using both its $\mathrm{J} 3$ and $\mathrm{W} 2$ photometry. The mean correction between $J_{\mathrm{MKO}}$ and $\mathrm{J} 3$ for cool dwarfs $\left(J_{\mathrm{MKO}}-\mathrm{J} 3=0.20 \pm 0.03\right)$ and the median

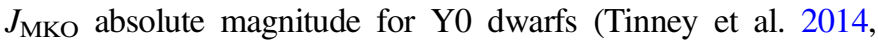
$\mathrm{M}_{J}=20.32 \pm 1.25$, giving a median $\left.M_{J 3}=20.12 \pm 1.25\right)$, gives a distance for W0302-5817 lying in the range 24-7.5pc. A better estimate can be obtained using W2 (the median absolute magnitude for a $\mathrm{Y} 0$ has much lower scatter- $\mathrm{M}_{\mathrm{W} 2}=$ $14.65 \pm 0.35$ ), which predicts $\mathrm{d}=17.5 \pm 3.5 \mathrm{pc}$. This places
W0302 on the outer edge of the 20 pc sample of nearby brown dwarfs.

W1141-3326 is the second new Y dwarf presented here. As noted earlier, despite being observed with $\mathrm{CH}_{4} \mathrm{~s}, \mathrm{CH}_{4} \mathrm{l}$ filters in mid-2012, it took some time to obtain spectra for this object, because confusion with background sources meant its $\mathrm{CH}_{4} \mathrm{~s}$ $-\mathrm{CH}_{4} \mathrm{l}$ color was that of a mid- $\mathrm{T}$ dwarf, so it was not made a high priority (see Figure 2). A high-quality spectrum was eventually obtained in 2014 March, and it is an excellent match to the template Y0 spectrum. W1141-3326's W2 photometry indicates a likely distance in the range $11.4-8.0 \mathrm{pc}$. This is a distance fully consistent with the preliminary trigonometric distance presented in Tinney et al. (2014) of $9.5 \pm 0.4 \mathrm{pc}$.

Both W1141-3326 and W0302-5817 are targets of our ongoing parallax program with FourStar on the Magellan Baade telescope (Tinney et al. 2014).

\subsection{Comparison with Extant Spectroscopy}

Table 10 compares previously published spectral types for objects where we present new spectra, as well as objects where we identify the object as a $\mathrm{T}$ dwarf but do not have spectroscopy. For W1433-0837 and W1448-2534, we obtain the same spectral types from completely independent spectra and typing processes, while for W2212-6931 we obtain a type that is different by only 0.5 sub-types, which we consider to be consistent-especially since an examination of Figure 8 in Schneider et al. (2015) suggests that W221-6931 has the narrowest $1.3 \mu \mathrm{m}$ peak of of all the T9 objects shown, and so is possibly the closest to T9.5 in that group on their classification system.

We have not been able to obtain a spectrum for W0404-6420, and the T9 classification of Schneider et al. (2015) is two sub-types later from that estimated by our $\mathrm{CH}_{4} \mathrm{~s}$ $-\mathrm{CH}_{4} \mathrm{l}$ photometry. This is 3-4 times larger than the 0.43-0.6 sub-type scatters observed in our calibration (Figure 4), which reinforces our view that while a methane absorption detection is robust for identifying a cool brown dwarf, and the color provides an estimate of the spectral type, spectroscopy remains the "gold standard" for a firm classification.

\section{Conclusion}

Our results show that, despite using a modest $4 \mathrm{~m}$ class telescope like the AAT on targets at $J \sim 20$, methane imaging is an effective technique for refining cool dwarf candidate lists arising from an external survey. These are magnitudes at which near-infrared spectroscopy on a $4 \mathrm{~m}$ telescope would be almost impossible, or at least prohibitively expensive. Methane imaging observations make both identifications and first estimates of the spectral type in a single observation-without having to obtain spectra for the multiple candidate targets that can usually be found in the substantial positional error boxes that arise from a survey like WISE.

Cool brown dwarfs from a large area, yet shallow survey, like WISE, will reside quite close to the Sun, and almost invariably have significant proper motions. As such, the position error box to search in a follow-up program grows with time. Indeed it has been our experience that substantial proper motions (i.e., <0!"2/year) are so ubiquitous for WISE brown dwarfs that once follow-up extended more than 
a few years beyond the baseline of the WISE mission, any objects that do positionally match with the WISE source to better than about half an arcsecond, are invariably found to not be cool brown dwarfs, but rather background sources.

We have shown that methane imaging observations-either in the $\mathrm{H}$-band using $\mathrm{CH}_{4} \mathrm{~S}$ and $\mathrm{CH}_{4} \mathrm{l}$ filters or in the J-band using $\mathrm{J} 2$ and $\mathrm{J} 3$ filters-can rapidly and efficiently identify and preliminarily classify cool brown dwarf candidates that arise from large, all-sky surveys like WISE.

We have presented data identifying 21 new $\mathrm{T}$ dwarfs and 2 new Y dwarfs from the WISE All-Sky Survey using methane imaging (in addition to the Y dwarf W1639 previously published by Tinney et al. 2012). In many cases, these identifications were made for objects using a $4 \mathrm{~m}$ class telescope (the AAT) for objects at $J \gtrsim 20$-magnitudes at which near-infrared spectroscopy on such a telescope would be either impossible or prohibitively expensive.

We present a further five late $\mathrm{T}$ dwarfs (W0309-5016, W0628-8057, W2017-3421, W2211-4758) and three early T dwarfs (W0042-5840, W2302-7134, W2354-8140) with methane identifications, for which typing spectroscopy is required.

C.G.T. gratefully acknowledges the support of ARC Australian Professorial Fellowship grant DP0774000 and ARC Discovery Outstanding Researcher Award DP130102695. We are grateful for the extraordinary support we have received from the AAT's technical staff-K. Fiegert, Y. Kondrat, S. Lee, R. Paterson, and D. Stafford. Australian access to the Magellan Telescopes was supported through the National Collaborative Research Infrastructure Strategy of the Australian Federal Government. Travel support for Magellan and AAT observing was provided by the Australian Astronomical Observatory.

This publication makes use of data products from the Widefield Infrared Survey Explorer, which is a joint project of the University of California, Los Angeles, and the Jet Propulsion Laboratory/California Institute of Technology, funded by the National Aeronautics and Space Administration. This publication also makes use of data products from 2MASS, which is a joint project of the University of Massachusetts and the Infrared Processing and Analysis Center/California Institute of Technology, funded by the National Aeronautics and Space Administration and the National Science Foundation. This research has made extensive use of the NASA/IPAC Infrared Science Archive (IRSA), which is operated by the Jet Propulsion Laboratory, California Institute of Technology, under contract with the National Aeronautics and Space Administration. This research has also benefitted from the $\mathrm{M}, \mathrm{L}$, and $\mathrm{T}$ dwarf compendium housed at DwarfArchives.org, whose server was funded by a NASA Small Research Grant, administered by the American Astronomical Society.

Facilities: AAT (IRIS2), Magellan:Baade (FourStar, FIRE), Keck:II (NIRSPEC), IRTF (SpeX), WISE, CTIO:2MASS.

Software: Figaro, FIREHOSE (http://www.mit.edu/people/ rsimcoe/FIRE/ob_data.htm), Spextool (Cushing et al. 2004), ORACDR (http://www.ukirt.hawaii.edu/instruments/cgs4/orac$\mathrm{dr} /$ printable.html).

\section{ORCID iDs}

C. G. Tinney (i) https://orcid.org/0000-0002-7595-0970 Jacqueline K. Faherty (i) https://orcid.org/0000-00016251-0573

Gregory N. Mace 주 https://orcid.org/0000-0001-7875-6391

Mike Cushing (1) https://orcid.org/0000-0001-7780-3352

Adam J. Burgasser (i) https://orcid.org/0000-0002-6523-9536

Scott S. Sheppard (1) https://orcid.org/0000-0003-3145-8682

Edward L. Wright (1) https://orcid.org/0000-0001-5058-1593

\section{References}

Bertin, E., \& Arnouts, S. 1996, A\&AS, 117, 393

Burgasser, A. J., Geballe, T. R., Leggett, S. K., Kirkpatrick, J. D., \& Golimowski, D. A. 2006, ApJ, 637, 106

Cardoso, C. V., Burningham, B., Smart, R. L., et al. 2015, MNRAS, 450, 2486 Cavanagh, B., Jenness, T., Economou, F., \& Currie, M. J. 2008, AN, 329, 295 Cushing, M. C., Kirkpatrick, J. D., Gelino, C. R., et al. 2011, ApJ, 743, 50

Cushing, M. C., Vacca, W. D., \& Rayner, J. T. 2004, PASP, 116, 362

Dupuy, T. J., Liu, M. C., \& Leggett, S. K. 2015, ApJ, 803, 102

Faherty, J. K., Beletsky, Y., \& Burgasser, A. J. 2014, ApJ, 790, 90

Herbst, T. M., Thompson, D., Fockenbrock, R., Rix, H.-W., \& Beckwith, S. V. W. 1999, ApJL, 526, L17

Horne, K. 1986, PASP, 98, 609

Kirkpatrick, J. D., Cushing, M. C., Gelino, C. R., et al. 2011, ApJS, 197, 19 Kirkpatrick, J. D., Cushing, M. C., Gelino, C. R., et al. 2013, ApJ, 776, 128 Kirkpatrick, J. D., Gelino, C. R., Cushing, M. C., et al. 2012, ApJ, 753, 156 Kirkpatrick, J. D., Kellogg, K., Schneider, A. C., et al. 2016, ApJS, 224, 36 Kirkpatrick, J. D., Schneider, A. C., Fajardo-Acosta, S., et al. 2014, ApJ, 783,122

Liu, M. C., Michael, C., Dupuy, T. J., et al. 2012, ApJ, 758, 57

Lodieu, N., Burningham, B., Day-Jones, A., et al. 2012, A\&A, 548, A53

Lucas, P. W., Tinney, C. G., Burningham, B., et al. 2010, MNRAS, 408, L56 Luhman, K. L. 2014, ApJL, 786, L18

Luhman, K. L., Burgasser, A. J., \& Bochanski, J. J. 2011, ApJL, 730, L9

Luhman, K. L., \& Esplin, T. L. 2016, AJ, 152, 78

Mace, G. N., Cushing, M. C., Skrutskie, M., et al. 2013, ApJ, 726, 30

Mainzer, A., Bauer, J., Grav, T., et al. 2011, ApJ, 731, 53

Mainzer, A. K. McLean, \& Ian, S. 2003, ApJ, 597, 555

McLean, I. S., Becklin, E. E., Bendiksen, O., et al. 1998, Proc. SPIE, 3354, 566 McLean, I. S., Graham, J. R., Becklin, E. E., et al. 2000, Proc. SPIE, 4008, 1048 McLean, I. S., McGovern, M. R., Burgasser, A. J., et al. 2003, ApJ, 596, 561 Nakajima, T., Oppenheimer, B. R., Kulkarni, S. R., et al. 1995, Natur, 378, 463 Oppenheimer, B. R., Kulkarni, S. R., Matthews, K., \& Nakajima, T. 1995, Sci, 270,1478

Persson, S. E., Murphy, D. C., Smee, S., et al. 2013, PASP, 125, 654

Pinfield, D. J., Gromadzki, M., Leggett, S. K., et al. 2014, MNRAS, 444, 1931

Rayner, J. T., Toomey, D. W., Onaka, P. M., et al. 2003, PASP, 115, 362

Rosenthal, E. D., Gurwell, M. A., Ho, \& Paul, T. P. 1996, Natur, 384, 243

Schneider, A. C., Cushing, M. C., Kirkpatrick, J. D., et al. 2015, ApJ, 817, 112

Schneider, A. C., Cushing, M. C., Kirkpatrick, J. D., \& Gelino, C. R. 2016a, ApJL, 823, L35

Schneider, A. C., Greco, J., Cushing, M. C., et al. 2016b, ApJ, 804, 92

Simcoe, R. A., Burgasser, A. J., Bernstein, R. A., et al. 2008, Proc. SPIE, 7014, $7014 \mathrm{U}$

Simcoe, R. A., Burgasser, A. J., Bochanski, J. J., et al. 2010, Proc. SPIE, 7735, 773514

Skrutskie, M. F., Cutri, R. M., Stiening, R., et al. 2006, AJ, 131, 1163 Thompson, M. A., Kirkpatrick, J. D., Mace, G. N., et al. 2013, PASP, 125, 809

Tinney, C. G., Burgasser, A. J., Kirkpatrick, J. D., \& McElwain, M. W. 2005, AJ, 130, 2326

Tinney, C. G., Faherty, J. K., Kirkpatrick, J. D., et al. 2012, ApJ, 759, 60

Tinney, C. G., Faherty, J. K., Kirkpatrick, J. D., et al. 2014, ApJ, 796, 39

Tokunaga, A. T., Simons, D. A., \& Vacca, W. D. 2002, PASP, 114, 180

Vacca, W. D., Cushing, M. C., \& Rayner, J. T. 2003, PASP, 115, 389

Wright, E. L., Eisenhardt, P. R. M., Mainzer, A. K., et al. 2010, AJ, 140, 1868 